\title{
Fast computation of the spectral correlation
}

\author{
Jérôme Antoni, Ge Xin*, Nacer Hamzaoui \\ Laboratoire Vibrations Acoustique, Univ Lyon, INSA-Lyon, LVA EA677, F-69621 Villeurbanne, France
}

\section{A R T I C L E I N F O}

\section{Article history:}

Received 16 May 2016

Received in revised form 6 December 2016

Accepted 11 January 2017

Available online 3 February 2017

\section{Keywords:}

Cyclostationarity

Cyclostationary signals

Cyclic spectral analysis

Spectral correlation

Cyclic modulation spectrum

Fast spectral correlation

Condition monitoring

Bearing diagnosis

Nonstationary regime

\begin{abstract}
A B S T R A C T
Although the Spectral Correlation is one of the most versatile spectral tools to analyze cyclostationary signals (i.e. signals comprising hidden periodicities or repetitive patterns), its use in condition monitoring has so far been hindered by its high computational cost. The Cyclic Modulation Spectrum (the Fourier transform of the spectrogram) stands as a much faster alternative, yet it suffers from the uncertainty principle and is thus limited to detect relatively slow periodic modulations. This paper fixes the situation by proposing a new fast estimator of the spectral correlation, the Fast Spectral Correlation, based on the short-time Fourier transform (STFT). It proceeds from the property that, for a cyclostationary signal, the STFT evidences periodic flows of energy in and across its frequency bins. The Fourier transform of the interactions of the STFT coefficients then returns a quantity which scans the Spectral Correlation along its cyclic frequency axis. The gain in computational cost as compared to the conventional estimator is like the ratio of the signal length to the STFT window length and can therefore be considerable. The validity of the proposed estimator is demonstrated on non trivial vibration signals (very weak bearing signatures and speed varying cases) and its computational advantage is used to compute a new quantity, the Enhanced Envelope Spectrum.
\end{abstract}

(c) 2017 Elsevier Ltd. All rights reserved.

\section{Conventions}

Whereas the SC $S_{x}(\alpha, f)$ is a theoretical quantity, the ACP $S_{x}^{A C P}(\alpha, f)$, the CMS $S_{x}^{C M S}(\alpha, f)$, and the Fast-SC $S_{x}^{\text {Fast }}(\alpha, f)$ are three different estimators of the SC.

The connections between the spectral quantities handled in the paper are schemed in Fig. 1.

Definitions of the SC found in the literature may differ in the measurement units. The definition given here is such that for a signal with measurement units $U$, the SC has units $U^{2} / \mathrm{Hz}$. It is a one dimensional density of variable $f$. As a consequence, the particular case $\alpha=0$ returns the power spectral density, $S_{x}(0, f) \equiv S_{x}(f)$. Another definition of the SC is actually as a two dimensional density of variables $f$ and $\alpha$, with units $U^{2} / \mathrm{Hz}^{2}$ [1]. The power spectral density is then evaluated as $S_{x}(f)=\lim _{B \rightarrow 0} \int_{-B}^{B} S_{x}(\alpha, f) d \alpha$.

\section{Introduction}

Whether of mechanical or electrical nature, rotating machine signals are perfectly modelled by cyclostationary processes. The reason is that, due to the inherent operation of a machine, signals are produced by some periodic - or cyclic - mechanisms.

\footnotetext{
* Corresponding author.

E-mail addresses: jerome.antoni@insa-lyon.fr (J. Antoni), ge.xin@insa-lyon.fr (G. Xin).
} 


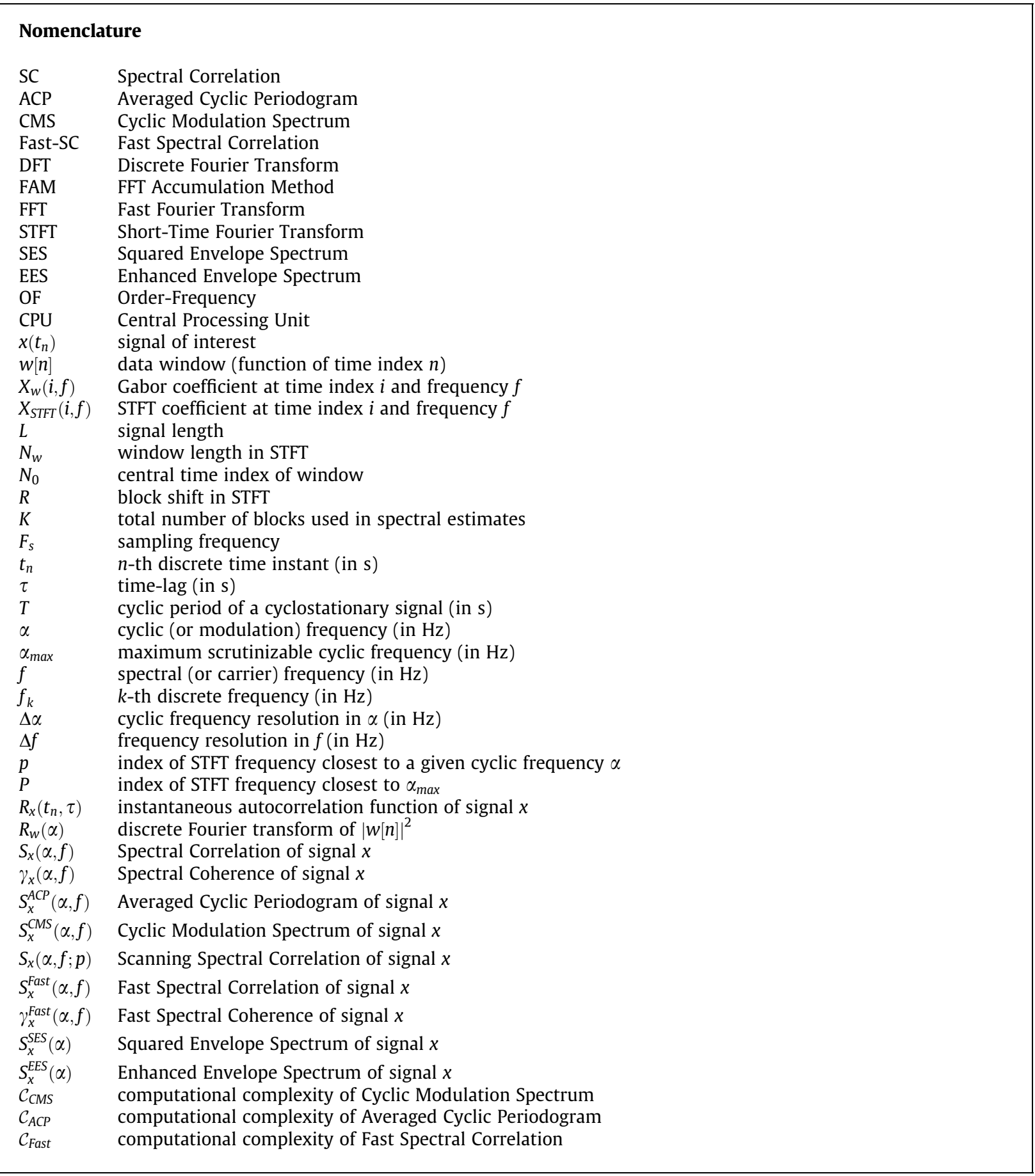

The cyclostationary class defines processes whose statistics are periodic. It encompasses most of the processes usually encountered in machines as particular cases, be they deterministic or random, e.g. periodic signals, stationary signals, periodically-modulated signals, repetitive transients, etc. This makes cyclostationarity a preferred framework in vibrationbased condition monitoring. Because of its ability to perfectly describe the statistical behavior of faults in the form of symptomatic modulations or repetition of transients, it provides optimal tools for their detection, their identification, and possibly their quantification. One central tool for the "cyclic spectral analysis" of machine signals is the Spectral Correlation (SC) which displays at once, in the form of a bi-spectral map, the whole structure of modulations and carriers in a signal [2-6]. Although the demonstration of the capabilities of cyclic spectral analysis in condition monitoring has been undertaken in several research works [7-13], its practice is still not as systematic as it deserves. Many methods are constantly published 


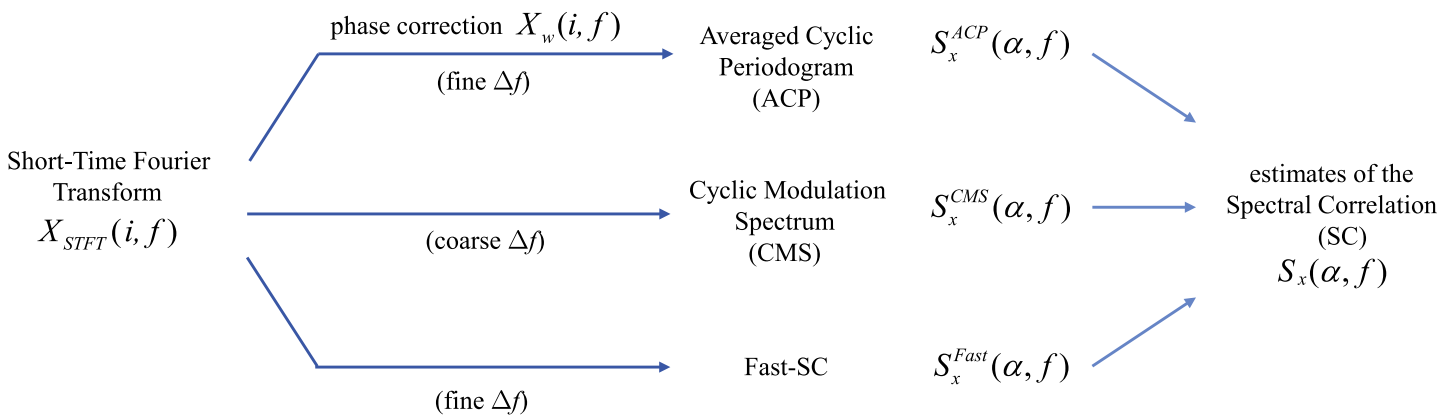

Fig. 1. Connections between the spectral quantities handled in the paper.

that painfully solve a diagnostic problem with sophisticated ad hoc tools where, instead, cyclostationarity would easily succeed. Two reasons might explain why a wider resort to cyclostationarity has been hindered so far. One is that it involves advanced theory of stochastic processes. Efforts have been spent to make this aspect as transparent as possible [14], but they are probably still insufficient. The second reason is that signal processing tools dedicated to cyclostationary analyses are so far computationally intensive. In particular, the SC may be extremely costly to compute in some situations, a fact which is likely to prevent its use by non-experts or for quick trouble shooting tasks. Alternatives have been proposed to the SC, like the Cyclic Modulation Spectrum (CMS) [15]. Being essentially a waterfall of envelope spectra at the output of a filterbank, it is fast to compute while intending to return similar information as the SC. The CMS has been recently formalized in Ref. [16] as an estimator of the SC. Although the CMS proves to be a valid diagnostic tool in many situations, it has limited performance in general: being constrained to the uncertainty principle, it cannot detect periodic patterns other than in the form of modulations whose frequencies are necessarily lower than the frequency resolution. As shown latter in this paper, the CMS is also not properly calibrated to quantify modulation depth. Besides, computationally efficient algorithms for the estimation of the SC have been proposed early in the nineteens in Refs. [3,17], of which the FFT Accumulation Method (FAM) is the fastest. As far as the authors known, the FAM is still recognized as the most computationally efficient algorithm in the specialized literature. Unfortunately, its computational advantage comes at the price of a degradation of the statistical performance of the estimator: the cyclic frequency resolution and variance are non-uniform, meaning that estimation errors can be locally very high; this is probably unacceptable in the kind of applications targeted by this paper.

The aim of this paper is to introduce a fast algorithm to estimate the SC, the "Fast Spectral Correlation" (Fast-SC), which essentially proceeds from the Short-Time Fourier Transform (STFT). It may be seen as a correction of the CMS such as to make it approach the ideal SC. Most of the computational effort is required for the calculation of the STFT - for which many efficient implementations now exist in commercial software. This makes the proposed algorithm weakly intrusive and of low complexity. For all these reasons, the approach proposed in this paper should participate in making the SC a more widely spread tool in condition monitoring.

Another contribution of this paper is to maintain a simple vision of cyclostationarity. While the CMS is simply interpreted as the detection of periodic flows of energy in frequency bands, the Fast-SC extends it to the detection of periodic flows across different frequency bands. This should help to make cyclostationarity easier to interpret while not sacrifying the usage of its most performant tools.

\section{Background on cyclic spectral analysis}

The object of this section is to resume the definitions and main properties of the SC and CMS; these will serve as a basis to discuss their pros and cons and will motivate the introduction of the Fast-SC in Section 3. Emphasis is also put on the two different visions entailed by the SC and the CMS in cyclic spectral analysis. The section opens with a presentation of the notations and main quantities used in the paper.

\subsection{Prerequisites}

Let denote the signal of interest $x\left(t_{n}\right)$ where $t_{n}=n / F_{s}$ refers to time instants acquired with sampling frequency $F_{s}$. Whenever convenient, the stream of samples $x\left(t_{n}\right), n=0, \ldots, L-1$, will be simply denoted by $x[n]$. The STFT of signal $x\left(t_{n}\right)$ over a time interval of length $N_{w} / F_{s}$ is defined as

$$
X_{\text {STFT }}\left(i, f_{k}\right)=\sum_{m=0}^{N_{w}-1} x[i R+m] w[m] e^{-j 2 \pi m_{F_{S}}^{f_{k}}}
$$

with discrete frequencies $f_{k}=k \Delta f, k=0, \ldots, N_{w}-1$, frequency resolution 


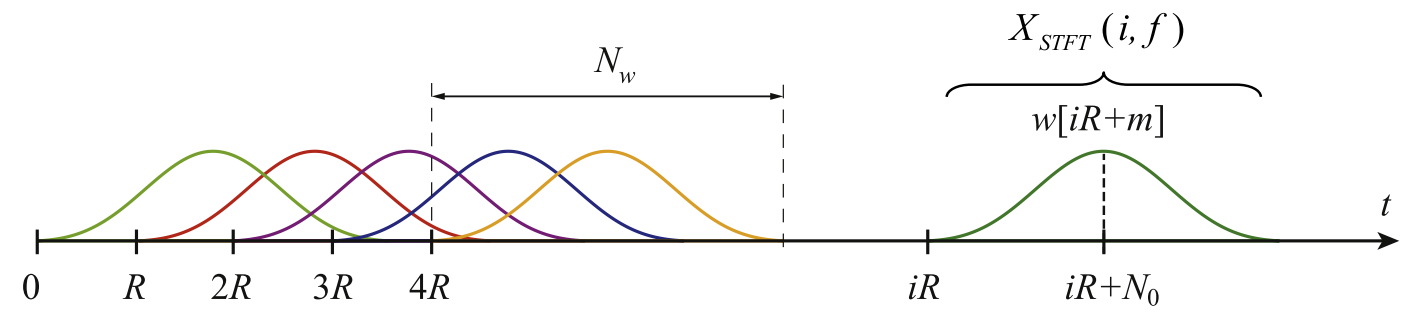

Fig. 2. Illustration of quantities $N_{w}, R$, and $N_{0}$ used in the Short-Time Fourier Transform.

$$
\Delta f=\frac{F_{s}}{N_{w}}
$$

a symmetric data window $w[m]$ with central time index $N_{0}$ such that $w\left[N_{0}+n\right]=w\left[N_{0}-n\right]\left(N_{0}=N_{w} / 2\right.$ if $N_{w}$ is even and $N_{0}=\left(N_{w}+1\right) / 2$ if it is odd), and time shift $R$ between consecutive windows - see Fig. 2.

In the subsequent analyses, the phase of the signal will play a fundamental role. It is therefore necessary to correctly reference it to the beginning of the signal, at the time origin $t_{n}=0$. This leads to the phase-corrected STFT (sometimes also presented as the Gabor transform), denoted here by

$$
X_{w}\left(i, f_{k}\right)=\sum_{n=0}^{L-1} x[n] w[n-i R] e^{-j 2 \pi n n_{\frac{f_{k}}{F_{S}}}}=X_{S T F T}\left(i, f_{k}\right) e^{-j 2 \pi i \frac{f_{k}}{F_{S}}}
$$

The interpretation of $X_{w}\left(i, f_{k}\right)$ is the "complex envelope" of signal $x[n]$ in a narrow frequency band of bandwidth $\Delta f$ centered on $f_{k}$ and sampled at time instants $i R / F_{s}$. Its squared magnitude, $\left|X_{w}\left(i, f_{k}\right)\right|^{2}$, thus reflects the energy flow in the frequency band [14]. The collection of squared coefficients $\left|X_{w}\left(i, f_{k}\right)\right|^{2}$ s for all time indices $i R$ and frequencies $f_{k}$ defines the spectrogram. From now on, signal $x\left(t_{n}\right)$ is assumed to be cyclostationary on the second-order. This means that its instantaneous autocorrelation function,

$$
R_{x}\left(t_{n}, \tau\right)=\mathbb{E}\left\{x\left(t_{n}\right) x\left(t_{n}-\tau\right)^{*}\right\},
$$

(where $\mathbb{E}$ stands for the ensemble average operator and ${ }^{*}$ for the complex conjugate) is a periodic function of time $t_{n}$ with some period $T$,

$$
R_{x}\left(t_{n}, \tau\right)=R_{x}\left(t_{n}+T, \tau\right)
$$

Intuitively, the periodicity of the autocorrelation function evidences the presence of a repetitive statistical behavior in the signal, for instance due to the occurrence of a fault in the form of a series of impulses or in the form of periodic modulations, but not only. The cyclostationary framework is actually rather large and includes many types of signals produced by periodic mechanisms.

The characterization of cyclostationary signals in the frequency domain usually provides more insight. This introduces the SC and its degraded version, the CMS.

\subsection{The Spectral Correlation}

The SC is defined as the double discrete Fourier transform of the instantaneous autocorrelation function (actually a Fourier series in time $t$ and a Fourier transform in time-lag $\tau$ when continuous time is considered),

$$
S_{x}(\alpha, f)=\lim _{N \rightarrow \infty} \frac{1}{(2 N+1) F_{s}} \sum_{n=-N}^{N} \sum_{m=-\infty}^{\infty} R_{x}\left(t_{n}, \tau_{m}\right) e^{-j 2 \pi n \frac{\alpha}{F_{s}}} e^{-j 2 \pi m \frac{f}{F_{s}}}, \quad t_{n}=\frac{n}{F_{s}}, \quad \tau_{m}=\frac{m}{F_{s}} .
$$

In the case of a second-order cyclostationary signal, the SC displays a characteristic signature continuous in frequency $f$ and discrete in cyclic frequency $\alpha$,

$$
S_{x}(\alpha, f)= \begin{cases}S_{x}^{k}(f), & \alpha=k / T \\ 0, & \text { elsewhere }\end{cases}
$$

where the $S_{x}^{k}(f)$ 's, $k=0, \pm 1, \pm 2, \ldots$ are "cyclic spectra”. In words, the SC is a two-dimensional representation (a bi-frequency map) made of a collection of parallel cyclic spectra at the discrete cyclic frequencies $\alpha=k / T$. The line at $\alpha=0$ returns the classical power spectral density.

The alignment of non-zero values at a given cyclic frequency $\alpha$ of the SC therefore indicates the existence of a sinusoidal modulation in the signal at that frequency $\alpha$, which envelops a carrier characterized by the cyclic spectrum $S_{x}^{k}(f)($ seen as a 
function of $f$ ). The SC may therefore be understood as a decomposition of the signal with respect to the "modulation frequency" $\alpha$ and the "carrier frequency" $f$.

A popular estimator of the SC is obtained from the so-called "time-smoothed cyclic periodogram" $[2,18]$ or, equivalently, the Averaged Cyclic Periodogram (ACP) [1] which is an extension of Welch's method (also known in spectral analysis as the "Weighted-Overlapped-Segment-Averaging" method) to cyclostationary signals. The ACP is defined as

$$
\begin{aligned}
& S_{x}^{A C P}(\alpha, f)=\frac{1}{K\|w\|^{2} F_{S}} \sum_{i=0}^{K-1} X_{w}(i, f) X_{w}(i, f-\alpha)^{*}, \\
& \|w\|^{2}=\sum_{n=0}^{N_{w}-1}|w[n]|^{2},
\end{aligned}
$$

where $X_{w}(i, f)$ is defined in Eq. (3) and $K=\left(L-N_{w}+R\right) / R$ is the total number of $N_{w}$-long windows shifted by $R$ samples in a $L$-long signal. One has [2]

$$
\lim _{N_{w} \rightarrow \infty K \rightarrow \infty} \lim _{x} S_{x}^{A C P}(\alpha, f)=S_{x}(\alpha, f)
$$

where lim denotes the probability limit taken in the mean-square sense.

One advantage of formula (8) is to express the SC as a measure of cross-correlation with respect to time between the complex envelopes at frequencies $f$ and $f-\alpha$. This highlights the property of a cyclostationary signal to be characterized by non-zero correlations between spectral components spaced apart by $\alpha$. In other words, spectral components at different frequencies are recruited synchronously in order to produce periodic modulations in the time domain.

The SC has been shown to be an ideal tool in condition monitoring because of its high capability to unwrap complicated signals onto a two-dimensional map that clearly reveals the presence of modulations and makes easy the identification of fault frequencies. It actually provides a high-resolution version of the envelope spectrum - an everlasting tool in condition monitoring - since the scrutinized carrier frequency band can be made arbitrarily small (the bandwidth is $\Delta f=F_{s} / N_{w}$ ) while still maintaining a very fine cyclic frequency resolution on the order of $\Delta \alpha=F_{s} / L$ (with $L$ the signal length). Despite all its advantages, the use of the SC may be hindered in practice by its high computational cost when large cyclic frequency ranges have to be explored (estimator (8) is computed in a loop over cyclic frequencies $\alpha_{k}=k \Delta \alpha$ ). This may be detrimental for unexperienced users, in particular when trials and errors approaches are followed.

In order to illustrate the estimation of the SC with the ACP, an example is given here of the cyclic spectral analysis of a simple cyclostationary signal. The signal is the response to white noise of an oscillator with a resonance frequency $f_{0}=250 \mathrm{~Hz}$ and damping ratio of $7 \%$, which is further modulated by a square wave with $90 \%$ dead time and frequency $\alpha_{0}=1.8 \mathrm{~Hz}$. The sampling frequency is $F_{s}=1000 \mathrm{~Hz}$ and the signal length is $L=10^{5}$ samples. The spectral correlation is computed from the ACP estimator of Eq. (8) with $N_{w}=2^{8}$, a Hann window, and $R=26$. This involves $K=3853$ windows and produces a frequency resolution $\Delta f=4 \mathrm{~Hz}$ and a cyclic frequency resolution $\Delta \alpha=0.01 \mathrm{~Hz}$. The estimated SC is displayed in Fig. 3(a) as a colormap. It is seen that the vertical lines are correctly identified at $\alpha_{0}$ and its multiples in a frequency band
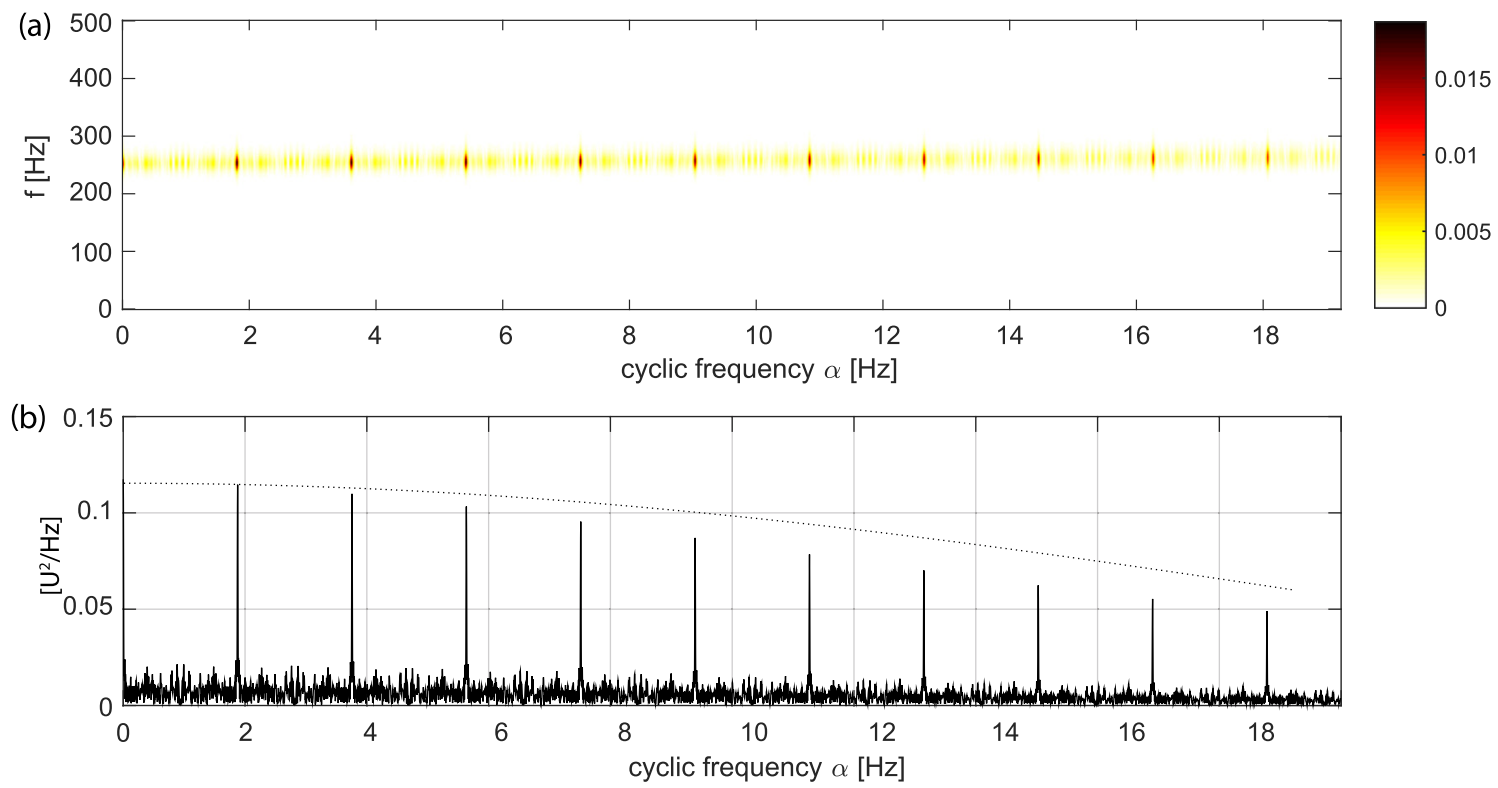

Fig. 3. (a) Spectral correlation estimated from the Averaged Cyclic Periodogram, $S_{x}^{A C P}(\alpha, f)$, with $N_{w}=2^{8}(\Delta f=4 \mathrm{~Hz} ; \Delta \alpha=0.01 \mathrm{~Hz})$ and (b) its evaluation at $f=250 \mathrm{~Hz}$ together with the theoretical envelope of the peaks (dotted line) as obtained from a square modulation. 
around the resonance at $250 \mathrm{~Hz}$. The SC evaluated at the resonance frequency, $S_{x}^{A C P}\left(\alpha, f_{0}\right)$, is displayed in Fig. 3(b): it is expected to show the Fourier spectrum of the square modulation whose theoretical envelope (the cardinal sine function) is indicated by the black dotted line. A slight underestimation is seen due to the picket fence effect (the multiples of $\alpha_{0}$ do not fall exactly on the cyclic frequency grid $\alpha_{k}=k \Delta \alpha$ ). It is worth noting that the computation of the ACP took more than 15 min on a laptop computer (i7-4810MQ Processor $2.80 \mathrm{GHz}$ ) which is detrimental as compared to other methods - more will be said on this matter in Section 4.

\subsection{The Cyclic Modulation Spectrum}

The CMS takes a different look at cyclostationary signals. It intends to track periodic flows of energy in frequency bands by evaluating the Fourier transform of the squared envelope at the output of a filter bank $[15,16]$. It is thus interpreted as a waterfall of envelope spectra. The CMS is efficiently computed as the Discrete Fourier Transform (DFT) of the spectrogram, i.e.

$$
\begin{aligned}
S_{x}^{C M S}(\alpha, f) & =\frac{1}{K\|w\|^{2} F_{S}} \sum_{i=0}^{K-1}\left|X_{\text {STFT }}(i, f)\right|^{2} e^{-j 2 \pi\left(i R+N_{0}\right) \frac{\alpha}{F_{S}}} \\
& =\frac{1}{K\|w\|^{2} F_{S}} \underset{i \rightarrow \alpha}{\operatorname{DFT}}\left\{\left|X_{\text {STFT }}(i, f)\right|^{2}\right\} e^{-j 2 \pi N_{0} \frac{\alpha}{F_{s}}},
\end{aligned}
$$

where it is reminded that $\left|X_{\text {STFT }}(i, f)\right|^{2}$ is an evaluation of the energy flow in a band centered on frequency $f$ and of bandwidth $\Delta f$ and where the notation DFT indicates that variable $i$ is transformed into $\alpha$. Note that the presence of $N_{0}$ in Eq. (10) is to evaluate the DFT at time instants $\left(i R+N_{0}\right) / F_{s}$ which corresponds to the centers of the windows of the STFT (see Fig. 2).

The computational cost of the CMS is essentially dictated by the computation of the STFT, for which very efficient implementation are nowadays available in most software. This makes it a very valuable tool in practice. Unfortunately, the CMS is a biased estimator of the SC and its approximation error increases with the cyclic frequency. It is shown in Appendix A that, provided that $N_{w}$ is longer than the correlation length of the signal (i.e. the extent of the instantaneous autocorrelation function along the $\tau$ axis),

$$
\mathbb{E}\left\{S_{x}^{C M S}(\alpha, f)\right\} \simeq S_{x}(\alpha, f) \frac{R_{w}(\alpha)}{R_{w}(0)}
$$

with

$$
R_{w}(\alpha)=\sum_{n=0}^{N_{w}-1}|w[n]|^{2} e^{-j 2 \pi\left(n-N_{0}\right) \frac{\alpha}{F_{s}}}
$$

(a)
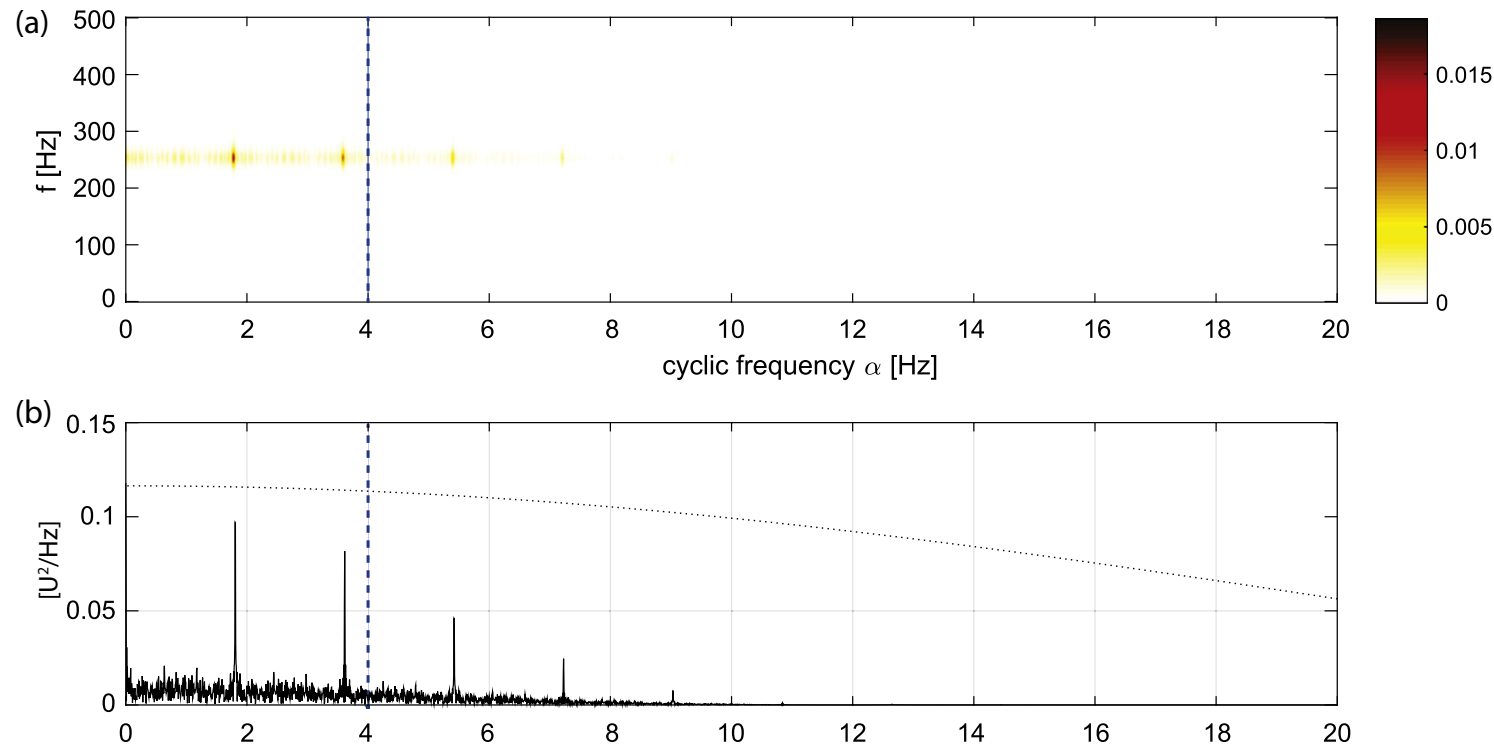

Fig. 4. (a) Cyclic Modulation Spectrum $S_{x}^{\text {CMS }}(\alpha, f)$ of the signal analyzed in Fig. 3 computed with $N_{w}=2^{8}$ and $R=26\left(\alpha_{\max } \sim \Delta f=4 \mathrm{~Hz}\right)$ and (b) its evaluation at $f=250 \mathrm{~Hz}$ together with the theoretical envelope of the peaks (dotted line) as obtained from a square modulation. The limit $\alpha_{\max }$ is indicated by the vertical blue dotted line. (For interpretation of the references to color in this figure legend, the reader is referred to the web version of this article.) 
and $R_{w}(0)=\|w\|^{2}$. Eq. (11) means that the CMS "sees" the SC through kernel $R_{w}(\alpha) / R_{w}(0)$. As shown in Fig. 6, the latter applies a low-pass weighting in the $\alpha$ direction with a cutoff frequency on the order of

$$
\alpha_{\max } \sim \frac{F_{s}}{N_{w}}=\Delta f
$$

In words, the STFT windows should not undersample the modulations in the signal.

As explained in Ref. [14], the fact that the highest cyclic frequency seen by the CMS is bounded upward by $\Delta f$ is a direct consequence of the uncertainty principle, $T \cdot \Delta f \geqslant 1$, which affects the STFT. With $T=1 / \alpha$, the latter reads

$$
\alpha \leqslant \Delta f
$$

Thus, modulations which are faster than allowed by the frequency resolution $\Delta f$ of the STFT are not detected. This subjects the CMS to a compromise between a fine resolution $\Delta f$ to accurately analyze the spectral content of the carrier and a coarse $\Delta f$ to enlarge the range $\alpha_{\max }$ of detectable modulation frequencies.

The limitation of the CMS is hereafter illustrated on the example of the previous subsection. Using the same parameters, the CMS is thus limited to detect modulations lower than $\alpha_{\max } \sim \Delta f=4 \mathrm{~Hz}$. This is evidenced in Fig. 4 where the cyclic upper limit is indicated by a vertical blue dotted line (the evaluation of the CMS at the resonance frequency, $S_{x}^{C M S}\left(\alpha, f_{0}\right)$, is shown in Fig. 7(b)). Obviously the limit could be pushed up by decreasing the value of $N_{w}$ at the detriment of frequency resolution $\Delta f$. However, it is advised to keep the latter reasonably fine in general in order to correctly identify the spectral content of the carrier in particular when several modulation patterns coexist in the $(\alpha, f)$ plane as is commonplace with complex vibration signals. Note that the computation of the CMS took only $1 \mathrm{~s}$.

(a)

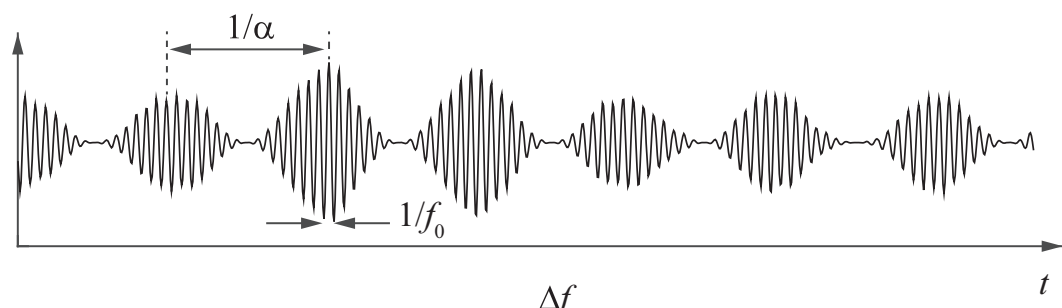

(b)

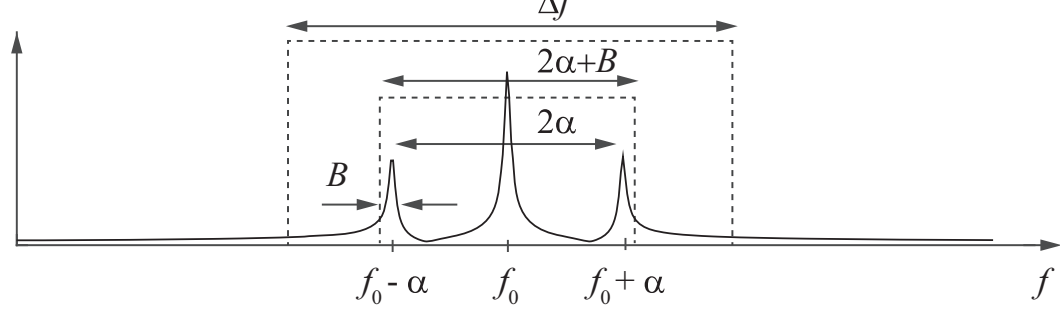

(c)

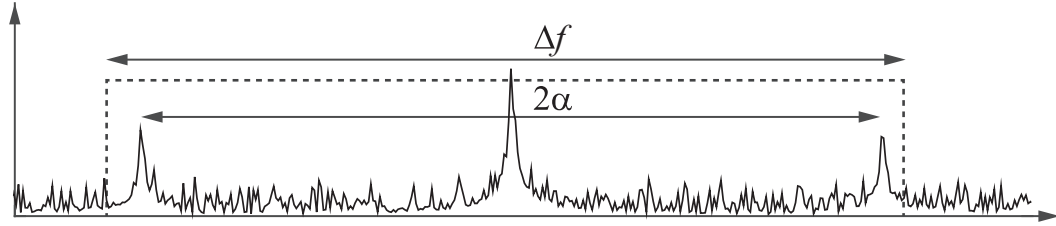

(d)

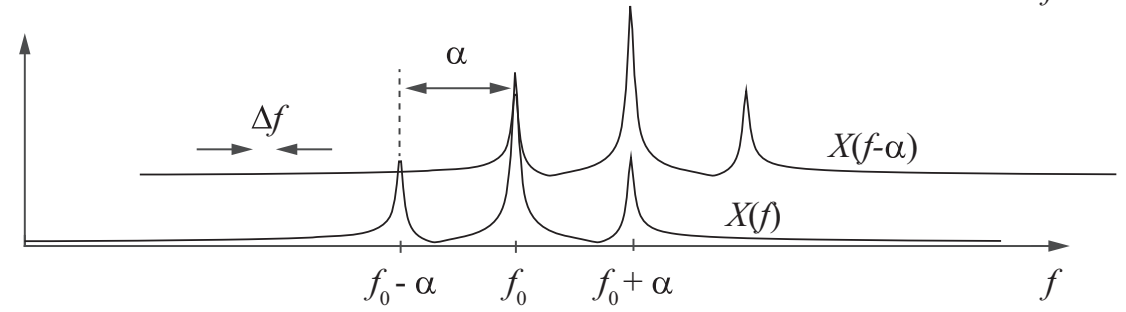

Fig. 5. (a) Example of a cyclostationary signal composed of a sinusoidal amplitude modulation on a narrow-band carrier and (b) its frequency spectrum. The CMS requires that $\Delta f>\alpha+B$ in order to fully capture the periodic beatings in time, which inevitably prevents a fine description of the spectral density. (c) Spectrum of a similar signal with faster modulation and presence of noise; the condition $\Delta f>\alpha+B$ implies that a large amount of noise is fatally absorbed in the analysis band. 


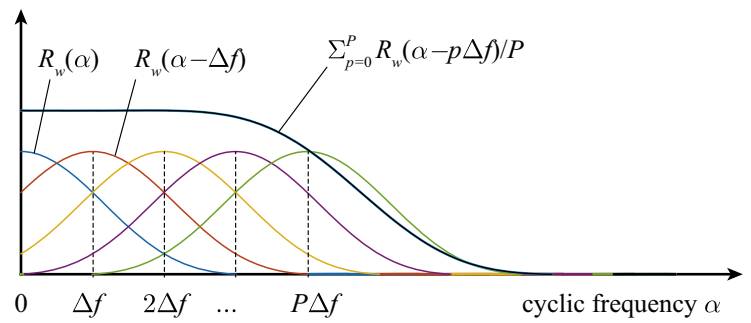

Fig. 6. Kernel function $R_{w}(\alpha)$, its shifted versions $R_{w}(\alpha-p \Delta f), p=1, \ldots, P$, and their sum (scaled by $1 / P$ ) used to equalize the Fast-SC in Eq. (24). The effective half-bandwidth of the aggregated kernel is about $P \Delta f=F_{s} /(2 R)$.

\subsection{Narrow-band modulations}

This subsection elaborates more on the type of cyclostationary patterns which the CMS can hardly characterize. Let consider the elementary modulated signal (chosen for the sake of simplicity),

$$
x\left(t_{n}\right)=a\left(t_{n}\right) \epsilon\left(t_{n}\right)
$$

where $\epsilon\left(t_{n}\right)$ stands for a stationary, narrow-band carrier with bandwidth $B$ centered on frequency $f_{0}$ and $a\left(t_{n}\right)=1+\cos \left(2 \pi \alpha t_{n}\right)$ is a periodic modulation with frequency $\alpha$. An example of such a signal and its corresponding spectrum are displayed in Fig. 5(a) in the case where $\alpha>B$.

Let us consider the Fourier transform $X(f)$ of signal $x\left(t_{n}\right)$ over a very long but finite time interval. It is easy to show that $X(f)=E(f)+\frac{1}{2} E(f-\alpha)+\frac{1}{2} E(f+\alpha)$ with $E(f)$ the Fourier transform of $\epsilon\left(t_{n}\right)$. For the CMS to detect the periodic modulation, its resolution $\Delta f$ must be large enough to contain the component $E(f)$ and its two shifted version $E(f-\alpha)$ or $E(f+\alpha)($ located around $f_{0}+\alpha$ and $f_{0}-\alpha$, respectively, in Fig. 5(b)) whose combination produces the characteristic periodic "beating" in the time domain. As seen in Fig. 5(b), this condition requires that $\Delta f>2 \alpha+B$, i.e. a frequency resolution $\Delta f$ for the CMS necessarily coarser than the bandwidth $B$ of the narrow-band carrier. As a result, the CMS is therefore unable to analyze accurately the spectral content of the carrier. The situation is particularly critical with fast modulations, $\alpha \gg B$, which imply that $\Delta f \gg B$. Such a condition is difficult to satisfy a priori when the signal to be analyzed is unknown. In addition, setting $\Delta f$ becomes particularly hazardous when the cyclic frequencies varies in time, a situation which is investigated in details in Section 6.3.

A last drawback of having to set $\Delta f$ exaggeratedly large as compared to the carrier bandwidth $B$ is to decrease the overall signal-to-noise ratio in the band and thus to lower the capability of detecting the presence of cyclostationarity. This is illustrated in Fig. 5(c) and further discussed in Section 6.1.3.

In contrast, the SC does not suffer from these limitations since it does not try to detect a periodic modulation of the signal energy in a band of width $\Delta f$, but rather searches for correlation between two frequency components at $f$ and $(f-\alpha)$. The frequency resolution $\Delta f$ can therefore be made arbitrarily fine to analyze accurately the spectral content of the carrier while still detecting the modulation. This is illustrated in Fig. 5(d).

\section{The Fast Spectral Correlation}

This section explains how the concept of the CMS can be extended, at moderate computational cost, to closely approach the ideal SC. The idea is first introduced on an intuitive ground and is next formally proved.

\subsection{An intuitive approach}

Let us start with the ACP estimator (8) of the SC and see how it can be expressed in terms of the STFT. The first constraint is to evaluate the spectral components at frequencies $f$ and $(f-\alpha)$ at multiples of $\Delta f=F_{s} / N_{w}$. Assuming that the constraint is satisfied for $f$, viz $f=f_{k}=k \Delta f$, an approximation is to be made for $\left(f_{k}-\alpha\right)$. It starts with the polar representation of the "wave packet"

$$
X_{w}\left(i, f_{k}-\alpha\right)=\left|X_{w}\left(i, f_{k}-\alpha\right)\right| e^{j \phi_{i}\left(f_{k}-\alpha\right)}
$$

by means of a magnitude and a phase. Each of these components is now going to be approximated in a different way. First, a rough approximation is allowed for the magnitude which smoothly envelopes the wave packet. Setting

$$
\alpha=p \Delta f+\delta
$$

where $p \Delta f$ is the closest frequency bin to $\alpha$ and $\delta$ the residue, it comes $\left|X_{w}\left(i, f_{k}-\alpha\right)\right| \simeq\left|X_{w}\left(i, f_{k-p}\right)\right|$. Second, a more accurate approximation is required for the phase since its derivative contains high frequency oscillations of the wave packet. Using a first-order Taylor expansion, $\phi_{i}\left(f_{k}-\alpha\right) \simeq \phi_{i}\left(f_{k}-p \Delta f\right)-\delta \cdot \phi_{i}^{\prime}\left(f_{k}-p \Delta f\right)$ where $\phi_{i}^{\prime}$ denotes the group delay of the wave packet. 
Since, by construction, the latter is located around time instant $\left(i R+N_{0}\right) / F_{s}$, it reads $\phi_{i}^{\prime}\left(f_{k}-p \Delta f\right) \simeq-2 \pi\left(i R+N_{0}\right) / F_{s}$ (see Fig. 2 and recall that, by definition, the group delay returns the time delay of a group of waves localized around a given frequency). Collecting all terms,

$$
\begin{aligned}
X_{w}\left(i, f_{k}-\alpha\right) & \simeq\left|X_{w}\left(i, f_{k-p}\right)\right| e^{j \phi_{i}\left(f_{k}-p \Delta f\right)} e^{j 2 \pi \frac{\delta}{F_{s}}\left(i R+N_{0}\right)} \\
& =X_{w}\left(i, f_{k-p}\right) e^{j 2 \pi \frac{\delta}{F_{s}}\left(i R+N_{0}\right)} \\
& =X_{w}\left(i, f_{k-p}\right) e^{j 2 \pi\left(\frac{\alpha}{F_{s}}-p \frac{\rho f}{F_{S}}\left(i R+N_{0}\right)\right.} .
\end{aligned}
$$

Now, inserting into Eq. (8) and using the correspondence (3) with the STFT, one arrives at

$$
\begin{aligned}
S_{x}\left(\alpha, f_{k} ; p\right) & =\frac{1}{K\|w\|^{2} F_{S}} \sum_{i=0}^{K-1} X_{w}\left(i, f_{k}\right) X_{w}\left(i, f_{k-p}\right)^{*} e^{-j 2 \pi\left(\frac{\alpha}{F_{S}}-\frac{p}{N_{w}}\right)\left(i R+N_{0}\right)} \\
& =\frac{1}{K\|w\|^{2} F_{S}} \sum_{i=0}^{K-1} X_{\text {STFT }}\left(i, f_{k}\right) X_{S T F T}\left(i, f_{k-p}\right)^{*} e^{-j 2 \pi \frac{\alpha}{F_{S}}\left(i R+N_{0}\right)} e^{j 2 \pi N_{N_{w}}^{p N_{w}}} \\
& =\frac{1}{K\|w\|^{2} F_{S}} \operatorname{DFT}_{i \rightarrow \alpha}\left\{X_{\text {STFT }}\left(i, f_{k}\right) X_{\text {STFT }}\left(i, f_{k-p}\right)^{*}\right\} e^{-j 2 \pi N_{0}\left(\frac{\alpha}{F_{S}}-\frac{p}{N_{w}}\right)}
\end{aligned}
$$

where the notation $S_{x}\left(\alpha, f_{k} ; p\right)$ is used to make the difference with the ACP estimator of Eq. (8) and to highlight the introduction of the frequency shift $p \Delta f$. The quantity $S_{x}\left(\alpha, f_{k} ; p\right)$ will be hereafter coined the "Scanning Spectral Correlation" for a reason to become clear shortly.

The above equation is a fundamental step towards the results of this paper. It suggests that the SC can be estimated simply from the Discrete Fourier Transform of the interactions between the STFT coefficients in frequency bins not necessarily spaced apart by exactly $\alpha$ (as required in the ACP). As a matter of fact, the CMS happens to be a particular case of $S_{x}(\alpha, f ; p)$ with $p=0$. It now remains to find how the quantity $S_{x}(\alpha, f ; p)$ is related to the theoretical SC and how a versatile and well calibrated estimator can be constructed from it.

\subsection{Scanning cyclic frequencies}

Taking the expected value of $S_{x}(\alpha, f ; p)$, it is shown in Appendix A that

$$
\mathbb{E}\left\{S_{x}(\alpha, f ; p)\right\} \simeq S_{x}(\alpha, f) \frac{R_{w}(\alpha-p \Delta f)}{R_{w}(0)}
$$

where $R_{w}(\alpha)$ is defined in Eq. (12). Kernel $R_{w}(\alpha-p \Delta f) / R_{w}(0)$ has effective bandwidth $2 \Delta f$ and is now centered on $p \Delta f$ (see Fig. 6).

The Scanning Spectral Correlation $S_{x}(\alpha, f ; p)$ scans a cyclic frequency zone roughly delimited by interval $[(p-1) \Delta f ;(p+1) \Delta f]$. This is illustrated in Fig. 7(b) when $p=4$ which is to be compared to Fig. 7(a) in the case $p=0$ corresponding to the CMS. It is seen that scanning provides a solution to scrutinize the cyclic frequency axis arbitrarily high without being limited any longer by the $\alpha_{\max } \sim \Delta f$ limit imposed to the CMS (see Section 2.3). By scanning the cyclic frequency axis with several values of $p$ and "merging" the corresponding intervals $[(p-1) \Delta f ;(p+1) \Delta f]$, the full SC can be reconstructed over the whole cyclic frequency axis, as illustrated in Fig. 7(c). This will define the "Fast Spectral Correlation", introduced hereafter in Section 3.4. Before proceeding further, the physical meaning of expression (20) is first discussed.

\subsection{Physical interpretation: periodic energy flow between STFT bins}

Eq. (19) is the DFT, evaluated at cyclic frequency $\left(\alpha / F_{s}-p / N_{w}\right)$, of the product of the STFT coefficient $X_{\text {STFT }}\left(i, f_{k}\right)$ and its frequency shifted version $X_{S T F T}\left(i, f_{k-p}\right)^{*}$. As a particular case, the CMS is returned for $p=0$, which reflects the fact that for a cyclostationary signal with cycle $T$, the energy $\left|X_{\text {STFT }}\left(i, f_{k}\right)\right|^{2}$ flows periodically in band $\left[f_{k}-\Delta f / 2 ; f_{k}+\Delta f / 2\right]$ with frequency $\alpha=1 / T$. Now, for $p \neq 0$, the interaction $X_{w}(i, f) X_{w}\left(i, f_{k-p}\right)^{*}$ measures the energy flow between bands $\left[f_{k}-\Delta f / 2 ; f_{k}+\Delta f / 2\right]$ and $\left[f_{k-p}-\Delta f / 2 ; f_{k-p}+\Delta f / 2\right]$. Using Eq. (7) one can show that (see Appendix A), on the average,

$$
\mathbb{E}\left\{X_{\text {STFT }}\left(i, f_{k}\right) X_{S T F T}\left(i, f_{k-p}\right)^{*}\right\} \simeq F_{s} \sum_{l} S_{x}^{l}(f) R_{w}\left(\alpha_{l}-p \Delta f\right) e^{j 2 \pi \frac{1}{I f_{s}}\left(N_{0}+i R\right)} e^{-j 2 \pi N_{N_{w}}^{p N_{0}}}
$$

with $S_{x}^{l}(f)$ as defined in Eq. (7). This proves that the interaction $X_{S T F T}\left(i, f_{k}\right) X_{S T F T}\left(i, f_{k-p}\right)^{*}$ contains a periodic function of time with frequency $\alpha=1 / T$. As a result, for a cyclostationary signal, the energy flows periodically between two STFT bins, with cyclic frequency $\alpha=1 / T$.

This is illustrated in Fig. 8 by a cyclostationary signal composed of a periodic amplitude modulation on a broad-band carrier. The real parts of the STFT coefficients in five adjacent frequency bins are displayed when the signal is "sampled" by a 

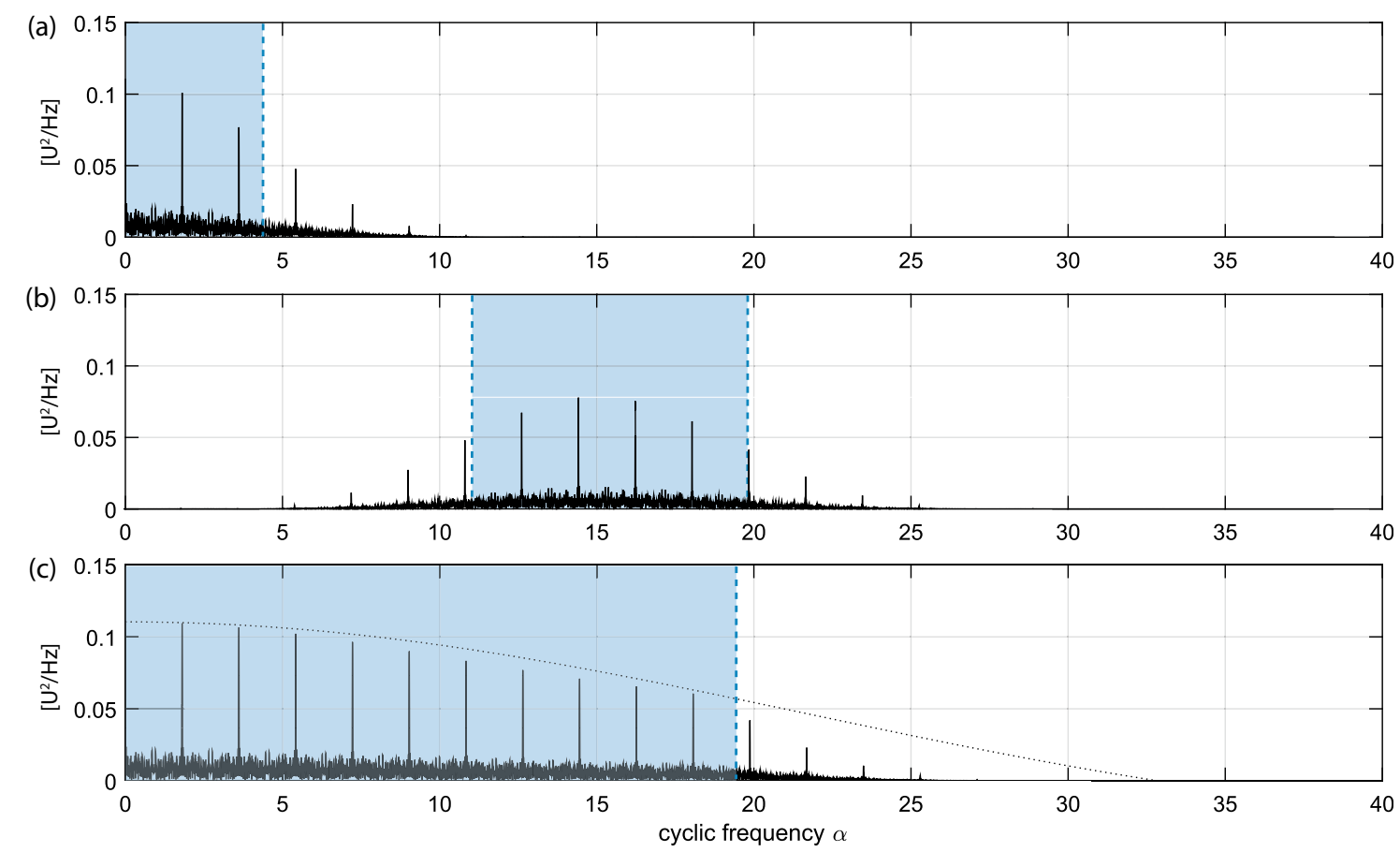

Fig. 7. (a) Cyclic Modulation Spectrum $S_{x}^{C M S}(\alpha, f)$ of the signal analyzed in Fig. 3, (b) scanning spectral correlation $S_{x}(\alpha, f ; p), p=4$, and (c) Fast Spectral Correlation $S_{x}^{\text {Fast }}(\alpha, f)$ all evaluated at $f=250 \mathrm{~Hz}$. The blue shaded areas indicate the effective cyclic range of each quantity. The black dotted line in (c) is the theoretical envelope of the peaks as obtained from a square modulation. (For interpretation of the references to color in this figure legend, the reader is referred to the web version of this article.)

(a)

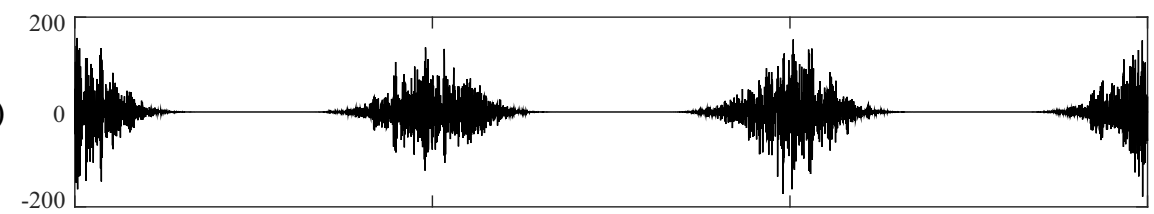

(b)

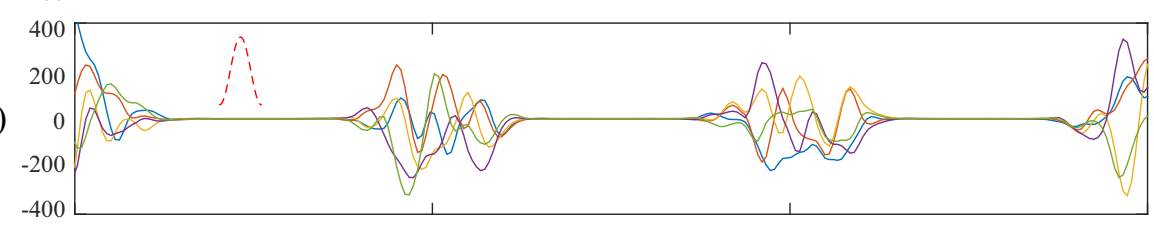

(c)

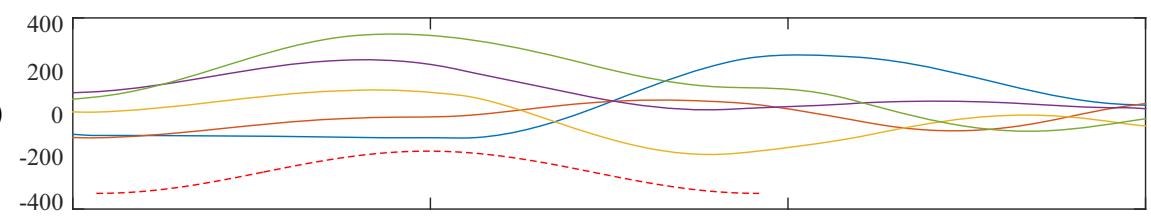

Fig. 8. (a) Example of a cyclostationary signal composed of a sinusoidal amplitude modulation on a broad-band noise carrier. (b) Real parts of the STFT coefficients $X_{\text {STFT }}\left(i, f_{k}\right)$ in five adjacent frequency bins $f_{k}, k=1, \ldots, 5$, in the case of a short window $w[n]$ (red dotted shape), such that $\Delta f>\alpha$. (c) Real parts of STFT coefficients in five adjacent frequency bins in the case of a long analysis window $w[n]$ (red dotted shape), such that $\Delta f<\alpha$. In (b), the presence of cyclostationarity is essentially reflected by the periodicity of the energy flow $\left|X_{\text {STFT }}\left(i, f_{k}\right)\right|^{2}$ in each subband, whereas in (c) it is encoded in the interaction $X_{\text {STFT }}\left(i, f_{k}\right) X_{\text {STFT }}\left(i, f_{l}\right)^{*}$ between STFT coefficients from different subbands $k \neq l$. (For interpretation of the references to color in this figure legend, the reader is referred to the web version of this article.)

short window $w[n]$ (Fig. 8(b)) and a long window (Fig. 8(c)). The first case is representative of $\Delta f>\alpha$ and visually evidences the periodicity of the energy flow (squared STFT coefficients) in each subband. The second case is representative of $\Delta f<\alpha$ and, as a consequence, hardly evidences periodicity of the energy flow. However, the presence of cyclostationarity is encoded in the correlation between the STFT coefficients coming from different subbands. 


\subsection{Definition of the Fast Spectral Correlation}

The fact that quantity $S_{x}(\alpha, f ; p)$ scans the SC in a cyclic frequency interval $[(p-1) \Delta f ;(p+1) \Delta f]$ naturally suggests its aggregation for several values of $p$ so as to reconstruct the SC over the whole cyclic frequency range. Given the sampling period $R / F_{s}$ of the STFT in Eq. (1), the maximum cyclic frequency that can be scanned is now

$$
\alpha_{\max } \sim \frac{F_{s}}{2 R},
$$

which corresponds to the maximum value of $p$,

$$
P=\left[\frac{F_{s} /(2 R)}{\Delta f}\right]=\left[\frac{N_{w}}{2 R}\right],
$$

where $[x]$ stands for the nearest whole number rounded down. This defines the "Fast Spectral Correlation" (Fast-SC)

$$
S_{x}^{\text {Fast }}(\alpha, f)=\frac{\sum_{p=0}^{P} S_{x}(\alpha, f ; p)}{\sum_{p=0}^{P} R_{w}(\alpha-p \Delta f)} R_{w}(0) .
$$

Note that the division by $\sum_{p=0}^{P} R_{w}(\alpha-p \Delta f)$ - which can be readily computed for a given data window $w[n]$ - equalizes the estimator from the effect of kernel $R_{w}(\alpha)$. It is proved in Appendix A that

$$
\mathbb{E}\left\{S_{x}^{\text {Fast }}(\alpha, f)\right\} \simeq S(\alpha, f)
$$

with asymptotic equality when $K \rightarrow \infty$ and $N_{w} \rightarrow \infty$. In addition, it is proved in Appendix C that the variance of the Fast-SC is

$$
\operatorname{Var}\left\{S_{x}^{F a s t}(\alpha, f)\right\} \simeq \frac{1}{K} \sum_{k \in \mathbb{K}} K_{w}\left(\alpha_{k}, \alpha\right) S_{x}^{k}(f) S_{x}^{k}(f-\alpha)^{*},
$$

almost everywhere, where $K_{w}\left(\alpha_{k}, \alpha\right)$ behaves like $R_{w}\left(\alpha_{k}\right)^{2} /\left(N_{w}^{2} w\left[N_{0}\right]^{4}\right)$. Therefore, combining the above two results, it holds that

$$
\lim _{N_{w} \rightarrow \infty K \rightarrow \infty} \lim _{x}^{\text {Fast }}(\alpha, f)=S_{x}(\alpha, f)
$$

(where the probability limit is taken in the mean-square sense) as for the ACP in Eq. (9). It is noteworthy that the expression of the variance of the Fast-SC has the same structure as that of the quadratic estimators of the SC (including the ACP) investigated in Ref. [1]. It is also proved in Appendix B that the frequency resolution of the Fast-SC is

$$
(\Delta f)_{\text {Fast }} \sim \frac{F_{s}}{N_{w}}
$$

for the carrier frequency and

$$
(\Delta \alpha)_{\text {Fast }} \sim \frac{F_{s}}{L}
$$

for the cyclic frequency, which is again in perfect accordance with other quadratic estimators of the SC (including the ACP) reported in [1].

The above results prove that the Fast-SC is an asymptotically convergent (unbiased and nil variance) estimator of the SC with similar statistical performance as the ACP. Its main advantage is that the computational effort is considerably alleviated as compared to the ACP (8) since it essentially relies on calculating the FFT of STFT products. It will be shown in the next section (Section 4) that it is also faster than the FAM [18], yet being closer to it than to the ACP in terms of computational complexity. However, contrary to the FAM, the Fast-SC does not suffer from non-uniform frequency resolution and has a uniformly bounded variance in the $(f, \alpha)$ plane.

Proceeding with the same example, the Fast-SC is now computed with the same parameters as for the ACP and CMS; it is displayed in Fig. 9 together with its evaluation at $250 \mathrm{~Hz}$. A comparison in three dimensions with the ACP and CMS is also displayed in Fig. 10. The computation took less than $5 \mathrm{~s}$, which is a considerable gain as compared to the ACP (15 min). It is seen that the maximum cyclic frequency has been pushed up much higher than the theoretical limit $\alpha_{\max } \sim 19 \mathrm{~Hz}$ of the CMS given by Eq. (22).

Fig. 7(c) shows that the theoretical magnitude of the peaks is accurately estimated and even slightly better than in the ACP of Fig. 3(b). This is because the Fast-SC is so fast as compared to the latter that zero-padding could be used in evaluating $S_{x}(\alpha, f ; p)$ by the DFT so as to increase the numerical resolution of the cyclic frequency $\alpha$ and thus reduce the picket fence effect. 

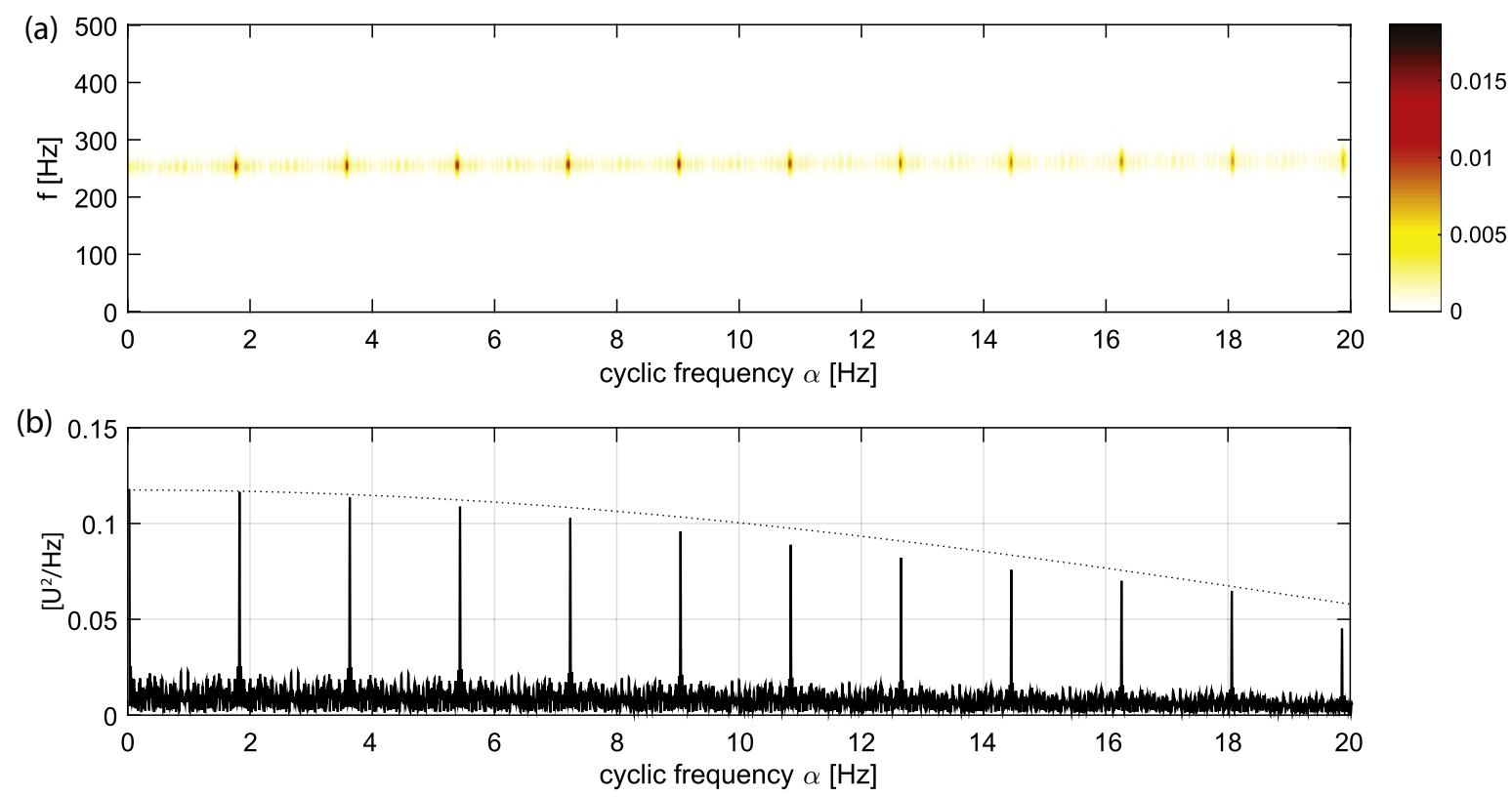

Fig. 9. (a) Fast Spectral Correlation $S_{x}^{\text {Fast }}(\alpha, f)$ of the signal analyzed in Fig. 3 computed with $N_{w}=2^{8}, R=26\left(\alpha_{\text {max }} \sim \Delta f=4 \mathrm{~Hz}\right)$ and $100 \%$ zero-padding and (b) its evaluation at $f=250 \mathrm{~Hz}$ together with the theoretical envelope of the peaks (dotted line) as obtained from a square modulation.
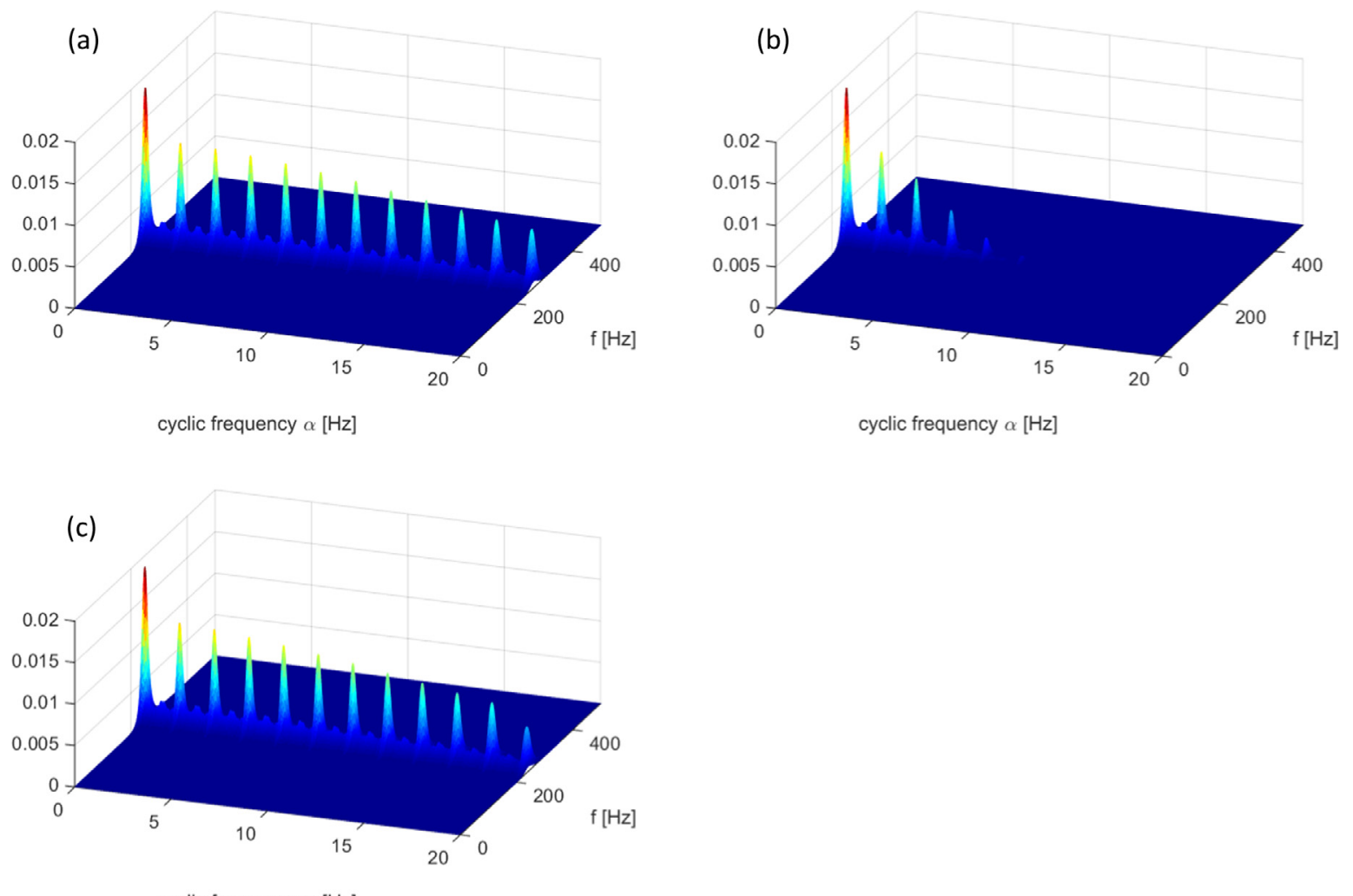

cyclic frequency $\alpha[\mathrm{Hz}]$

Fig. 10. 3D comparison of estimates of the SC obtained from (a) the ACP, (b) the CMS and (c) the Fast-SC. 


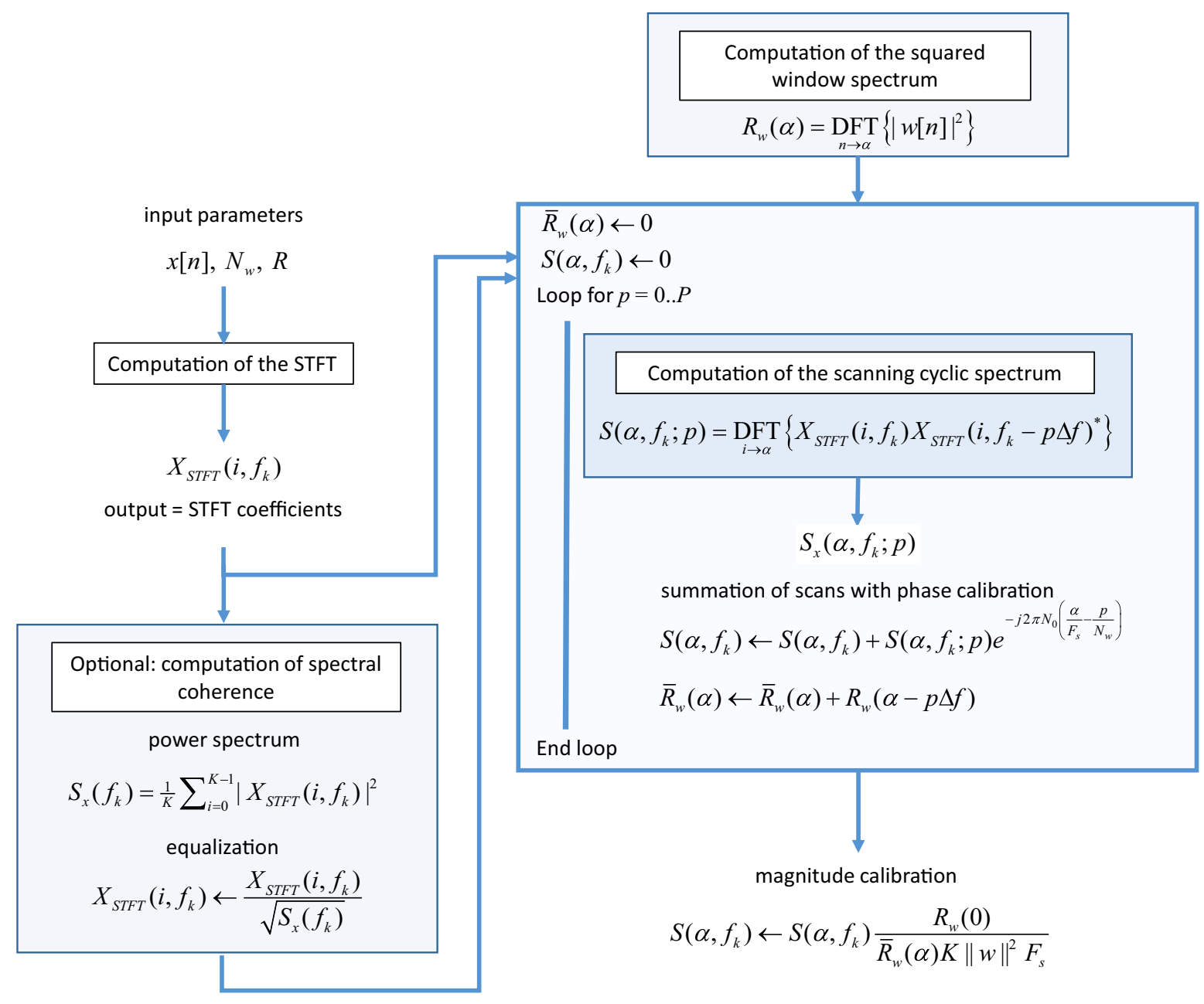

Fig. 11. Block-diagram of the Fast-SC algorithm.

\subsection{The Fast-SC algorithm}

The block-diagram of the Fast-SC algorithm is given in Fig. 11. It is seen that the implementation of the Fast-SC is easily amenable to parallel computing, which allows further potential speedup. A publicly available version of the algorithm coded in Octave/Matlab has been posted at the following address: https://fr.mathworks.com/matlabcentral/fileexchange/60561.

\section{Computational cost}

The computational cost of the Fast-SC is now addressed and compared to that of the ACP, the CMS and the FAM. For the sake of simplicity and because it will make possible a direct comparison with the results published in Ref. [18], the computational cost is addressed in terms of the number of complex multiplications. In addition, the same complex-valued FFT algorithm is considered in all cases (independently of whether the processed sequences are actually real or complex), i.e. with a fixed computational complexity on the order of $N \log _{2} \mathrm{~N}$ for a $\mathrm{N}$-long sequence.

\subsection{Computational cost of the Fast-SC}

For the Fast-SC, the count starts with data tapering of $K$ blocks of $N_{w}$ samples each, that is a complexity $K N_{w}$. Next, the calculation of the STFT involves the DFT of $K$ blocks of data of length $N_{w}$. Assuming that the Fast Fourier Transform (FFT) is used ( $N_{w}$ is a power of two), the complexity is like $K N_{w} \log _{2}\left(N_{w}\right)$. Next, there are $K$ products of $\left(N_{w} / 2-p\right)$ STFT coefficients repeated for $p=0, \ldots, P$ (only positive frequencies are considered due to symmetry of the FFT and shifted frequencies outside this range are not considered to avoid aliasing), which amounts to a complexity $K\left(N_{w} / 2+\left(N_{w} / 2-1\right)+\cdots+\right.$ $\left.\left(N_{w} / 2-P\right)\right)=K((P+1) / 2)\left(N_{w}-P\right)$. Finally, the products of $\left(N_{w} / 2-p\right)$ STFT coefficients are Fourier transformed over $K$ 
blocks for $p=0, \ldots, P$, leading to a complexity on the order of $((P+1) / 2)\left(N_{w}-P\right) K \log _{2}(K)$. Ignoring other computations such as calibration, phase correction and the equalization in Eq. (24), the complexity is

$$
\mathcal{C}_{\text {Fast }} \sim K N_{w}\left(1+\log _{2}\left(N_{w}\right)+\frac{P+1}{2}\left(1-\frac{P}{N_{w}}\right)\left(1+\log _{2}(K)\right)\right) .
$$

Considering that $K \sim L / R$ and $P \sim N_{w} /(2 R)$, the complexity eventually reads

$$
\mathcal{C}_{\text {Fast }} \sim \frac{L N_{w}}{2 R}\left(2+2 \log _{2}\left(N_{w}\right)+\left(\frac{N_{w}}{2 R}+1\right)\left(1-\frac{1}{2 R}\right)\left(1+\log _{2}\left(\frac{L}{R}\right)\right)\right) .
$$

Of concern is now to compare this result with the complexity of other estimators.

\subsection{Computational cost of the CMS}

The complexity of the CMS comes as a particular case of Eq. (30) with $P=0$, that is

$$
\mathcal{C}_{\text {CMS }} \sim \frac{L N_{w}}{2 R}\left(3+2 \log _{2}\left(N_{w}\right)+\log _{2}\left(\frac{L}{R}\right)\right) .
$$

Therefore, the computational gain of the CMS over the Fast-SC is, asymptotically,

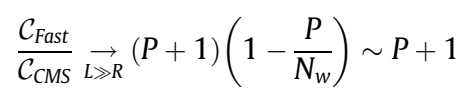

which is upper bounded by $P+1$.

\subsection{Computational cost of the ACP}

By comparison, the ACP in Eq. (8) computed over the same cyclic frequency range requires $\alpha_{\max } / \Delta \alpha=$ $\left(F_{s} /(2 R)\right) /\left(F_{s} / L\right) \sim L /(2 R)$ cyclic frequencies to be processed. The calculation of each cyclic frequency involves the tapering of the original signal and of its frequency shifted version for a complexity $2 K N_{w}$ and $K$ products of $N_{w} / 2$ STFT coefficients for a complexity $K N_{w} / 2$. Next comes the FFTs of $K$ blocks of $N_{w}$ samples for the original signal and of its frequency shifted version for a complexity of $2 K N_{w} \log _{2}\left(N_{w}\right)$. Ignoring other computations (e.g. multiplication by complex exponentials for the phase correction), the leading term in the overall complexity of the ACP is $\mathcal{C}_{A C P} \sim N_{w}(L /(2 R))^{2}\left(5+4 \log _{2}\left(N_{w}\right)\right)$. Therefore, the computational gain is

$$
\frac{\mathcal{C}_{A C P}}{\mathcal{C}_{\text {Fast }}} \sim \frac{L}{2 R} \underbrace{\left(\frac{5+4 \log _{2}\left(N_{w}\right)}{2+\log _{2}\left(N_{w}\right)+\left(\frac{N_{w}}{2 R}+1\right)\left(1-\frac{1}{2 R}\right)\left(1+\log _{2}\left(\frac{L}{R}\right)\right)}\right)}_{\rho} \underset{L \gg R}{\rightarrow} \frac{L}{2 R} .
$$

Since factor $\rho$ in the above equation is mainly located between 0.5 and 2 for wide ranges of values of $N_{w}$ and $L$ (e.g. $2^{3} \leqslant N_{w} \leqslant 2^{12}$ and $2^{10} \leqslant L \leqslant 2^{20}$ with $R=N_{w} / 4$ ), the asymptotic result $\mathcal{C}_{A C P} / \mathcal{C}_{\text {Fast }} \sim L / 2 R$ actually returns a very good approximation of the computational gain.

\subsection{Computational cost of the FAM}

The computational cost of the FAM has been worked out in Ref. [18]. Using the notation of the present paper and considering a complex-valued FFT algorithm, it reads

$$
\mathcal{C}_{\text {FAM }} \sim \frac{L N_{w}}{2 R}\left(4+\log _{2}\left(N_{w}\right)+\frac{N_{w}}{2}\left(1+\log _{2}\left(\frac{L}{R}\right)\right)\right) .
$$

The computational gain of the Fast-SC over the FAM is found bounded as

$$
1<\frac{\mathcal{C}_{\text {FAM }}}{\mathcal{C}_{\text {Fast }}}<R
$$

with asymptotic behavior

$$
\frac{\mathcal{C}_{\text {FAM }}}{\mathcal{C}_{\text {Fast }}} \underset{L \gg R}{\longrightarrow} \frac{R}{\left(1+\frac{2 R}{N_{w}}\right)\left(1-\frac{1}{2 R}\right)} .
$$

For a fixed ratio $R / N_{w}$ (as actually advocated in Ref. [18]), this is found to be on the order of $R$. 


\subsection{Discussion}

The former results indicate the following ranking of computational complexities,

$$
\mathcal{C}_{\text {CMS }} \leqslant \mathcal{C}_{\text {Fast }}<\mathcal{C}_{\text {FAM }}<\mathcal{C}_{\text {ACP }}
$$

with computational gains which can be roughly summarized as

$$
\frac{\mathcal{C}_{\text {Fast }}}{\mathcal{C}_{\text {CMS }}} \sim P+1, \quad \frac{\mathcal{C}_{\text {FAM }}}{\mathcal{C}_{\text {Fast }}} \sim R, \quad \text { and } \quad \frac{\mathcal{C}_{\text {ACP }}}{\mathcal{C}_{\text {Fast }}} \sim \frac{L}{2 R}
$$

when $L \gg R$.

Several conclusions are noteworthy at this juncture.

- Without surprise, the CMS has the lowest complexity, yet the Fast-SC comes close to it when the cyclic frequency range to scan is limited - i.e. when $P$ is small. The Fast-SC boils down to the CMS when $P=0$.

- The Fast-SC has a lower complexity than the FAM and its computational gain with respect to latter is found always greater than one. In particular, by taking the condition $R=N_{w} / 4$ which is recommended for the FAM in Ref. [18], it can be verified from Eq. (37) that $\mathcal{C}_{\text {FAM }} / \mathcal{C}_{\text {Fast }} \sim N_{w} / 6$.

- The computational gain of the Fast-SC over the ACP grows proportionally with the signal length $L$, which is the most considerable gain of all.

- Keeping in mind that $R$ is a fraction of $N_{w}$, the gain over the ACP is seen directly related to the variance reduction factor $L / N_{w}$, a quantity that reflects the quality of the SC estimation [1,14]. In practice, the latter should be as large as possible (e.g. typically more than a few tens of hundreds). This proves that in any scenario where the SC is to be estimated with a small estimation variance, the Fast-SC will have a computational advantage over the ACP.

- One exception where the ACP estimator remains advantageous is when the SC is estimated for one or a few cyclic frequencies $\alpha$ only.

Some of these conclusions are now verified by means of a numerical experiment. Fig. 12 compares the CPU (Central Processing Unit) time (minimum on 100 runs) required to compute the CMS, the Fast-SC and the ACP on a laptop computer (i7-4810MQ Processor $2.80 \mathrm{GHz}$ ). The signal is arbitrarily generated a hundred times (the computation time is supposed not to depend on the signal structure) and processed for different lengths $L$ ranging from $2^{10}$ to $2^{21}$. The window length is set arbitrarily to $N_{w}=2^{6}$, which is small enough to make possible the calculation of the ACP of long signals. A Hann window

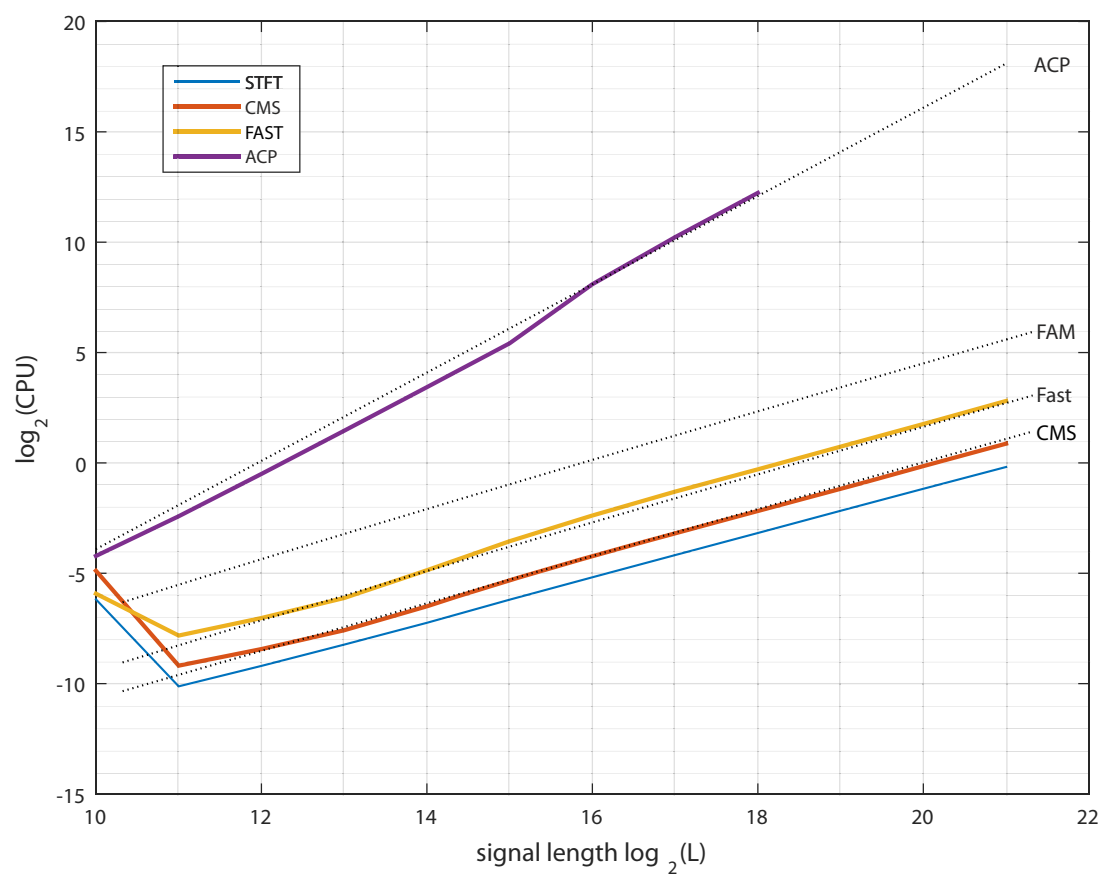

Fig. 12. CPU time in seconds (minimum value over 100 runs) required to compute the STFT, the Cyclic Modulation Spectrum (CMS), the Fast Spectral Correlation (Fast-SC) and the Averaged Cyclic Periodogram (ACP) for different signal lengths $L$ with $N_{w}=2^{6}$ and $R=10$. The dotted black line shows the theoretical CPU time of the ACP. 
is used with shift $R=10$ which corresponds to nearly $85 \%$ overlap. Also shown is the CPU time required to compute the STFT. For the CMS and Fast-SC, care is taken to use the next power of two for $K$ in formula (19) in order to benefit from the fastest version of the FFT. In addition, the time required to compute the denominator in Eq. (24) (used for calibration) is not accounted for - this factor can actually be pre-calculated.

Comparison of the results with the theoretical trends shows good agreement for signal lengths larger than $2^{11}$. It is believed that the departure for smaller values of $L$ is due to the time used for memory allocation (not accounted for in the above results) which prevails on short signals. The asymptotic behavior for long signals is yet very good. It is seen that the gain in computational time becomes considerable when the signal length increases: for instance when $L=2^{17}$, the CPU time is about $0.3 \mathrm{~s}$ for the Fast-SC whereas it is $10 \mathrm{~min}$ for the ACP; when $L=2^{20}$ (which is not uncommon in practice), the $\mathrm{CPU}$ time is $3.7 \mathrm{~s}$ for the Fast-SC whereas it is about 17.7 hours for the ACP (not computed in the experiment!).

\section{Practical recommendations}

Just as for any spectral quantity, the estimation of the SC requires careful setting of some parameters. In the case of the Fast-SC, the number of parameters to tune can be reduced to two: the maximum cyclic frequency to scrutinize, $\alpha_{\max }$, and the frequency resolution, $\Delta f$, of the carrier. According to formulas (2) and (22), they correspond to setting $N_{w}$ and $R$, respectively. The investigation of the computational cost undertaken in the former section will now help to provide strait guidelines to set the values of $R$ and $N_{w}$.

\subsection{Setting the block shift $R$}

The value of the block shift $R$ governs the maximum cyclic frequency $\alpha_{\max }$ that can be scrutinized according to Eq. (22). At the same time, $R$ should not be taken too small since computational cost was found inversely proportional to it in Eq. (31). Yet, it has been shown in Ref. [1] that a significant fraction of overlap should be provisioned to avoid cyclic leakage (a recommended value is at least $75 \%$ with classical windows, that is $R \sim 0.25 N_{w}$ ). Therefore, a safe guideline is to choose the greatest value which complies with the constraint

$$
R \leqslant \min \left(0.25 N_{w}, \frac{F_{s}}{2 \alpha_{\max }}\right) \text {. }
$$

\subsection{Setting the window length $N_{w}$}

The value of the window length $N_{w}$ governs the frequency resolution $\Delta f$ according to Eq. (28). There are two reasons while $N_{w}$ should be taken as small as possible while not sacrifying the required spectral resolution. First, the computational complexity has been found proportional to the square of $N_{w}$ in Eq. (31). Second, it has been shown in Ref. [1] that in order to control estimation errors, any linear estimator of the SC should satisfy $\Delta f \gg \Delta \alpha$, that is $N_{w} \ll L$.

\section{Examples of application}

This section illustrates the use of the Fast-SC on several experiments concerned with the diagnostics of rolling element bearings. Several of the signals actually happen to be too long to be analyzed with the ACP in a reasonable time, a situation that only makes possible the use of the CMS and the Fast-SC.

As explained in Refs. $[9,11,19]$, a distinctive symptom of faulty rolling element bearings is to produce cyclostationary signals, both in the incipient and the advanced stages. This is materialized by an alignment of non-zero components in the SC at the corresponding fault frequency, $\alpha=f_{\text {fault }}$. The magnitude and the number of harmonics linked to the incriminated cyclic frequency may serve as an indicator of severity of the fault. As advocated in Ref. [11], the Spectral Coherence is computed instead of the SC. The Spectral Coherence

$$
\gamma_{x}(\alpha, f)=\frac{S_{x}(\alpha, f)}{\sqrt{S_{x}(f) S_{x}(f-\alpha)}}
$$

with $S_{x}(f) \equiv S_{x}(0, f)$ (as obtained from the time average of the spectrogram) is a normalized version of the SC with magnitude normalized within 0 and 1 . It may be directly interpreted as the "depth" of a modulation with frequency $\alpha$ and carrier $f$. The Spectral Coherence may also be interpreted as the SC of the whitened signal, which tends to equalize regions with very different energy levels and thus to magnify weak cyclostationary signals. In the following, the Spectral Coherence will serve as a basis to define the Squared Envelope Spectrum (SES) [9],

$$
S_{x}^{S E S}(\alpha)=\left|\int_{f_{1}}^{f_{2}} \gamma_{x}(\alpha, f) d f\right|,
$$




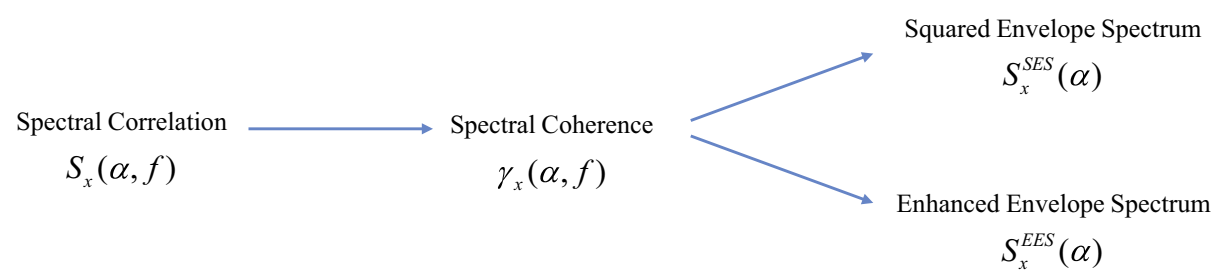

Fig. 13. Connection between the Spectral Correlation and the envelope spectra handled in the paper.

measured in a given frequency band $\left[f_{1} ; f_{2}\right]$ and, a newly proposed spectral quantity, the "Enhanced Envelope Spectrum" (EES)

$$
S_{x}^{E E S}(\alpha)=\int_{f_{1}}^{f_{2}}\left|\gamma_{x}(\alpha, f)\right| d f .
$$

The SES and the EES have the squared units of the signal. The integral in Eqs. (42) and (43) are replaced by discrete sums over frequencies $f_{k}=k \Delta f$ when its comes to estimators.

It is noteworthy that $S_{x}^{S E S}(\alpha) \leqslant S_{x}^{E E S}(\alpha)$ in general. The EES is expected to better enhance non-zero cyclic components than the SES because the latter integrates complex values, a process which may possibly converge towards zero in the case of fast rotating phases as demonstrated in Section 6.1.3.

The connection between the SC, the Spectral Coherence, the SES and the EES is schemed in Fig. 13. These quantities are now compared on case studies.

\subsection{Bearing signatures in fans}

This first experiment deals with the detection of rolling element signatures in small fans in a production line. Being brand new, the rolling element bearings are not expected to be seriously damaged, yet small defects due to possible mishandling or forces exerted during mounting are to be detected. This presents several challenges. First, the rolling element bearing signature is expected to be weak. Second, it happens to occur in very localized frequency bands, which asks for a fine frequency resolution $\Delta f$. Third, the number of harmonics has to be assessed accurately as it provides an indication of the fault severity; this requires scrutinizing a wide cyclic frequency range $\alpha_{\max }$. As explained in Section 2.3, these constraints are difficult to be satisfied conjointly by the CMS.

Vibration signals are recorded on fans hanged by tensioners in order to meet free-free boundary conditions. The sampling frequency is $F_{s}=131,072 \mathrm{~Hz}$ in case 1 and $F_{s}=51,200 \mathrm{~Hz}$ in cases 2 and 3 . The recording time is $5 \mathrm{~s}$. All signals are processed in the same way. First, the harmonics of the shaft rotation are removed - as advocated in Ref. [19] - with a combfilter. Next, the cyclic range $\alpha_{\max }$ is chosen so as to include at least 3 harmonics of the highest fault frequency, viz the Ball Pass Frequency on Inner Race (BPFI). This fixes the window length $N_{w}$ to be used with the CMS according to formula (13). The window length in the Fast-SC is set to $N_{w}=2^{9}$ in order to achieve a frequency resolution $\Delta f=100 \mathrm{~Hz}$. The block shift $R$ is set according to formula (22) in order to reach the required cyclic frequency range $\alpha_{\max }$. A Hann window is used. The parameter settings are reported in Table 1 for the three tested fans. In all cases, the SES and the EES are computed over the full frequency range $\left[f_{1}=0 ; f_{2}=F_{s} / 2\right]$ and hence referred to as wide-band envelope spectra.

\subsubsection{Case 1}

The first tested fan evidences a marked signature of the rolling elements. Fig. 14(a) and (b) show excerpts of the Spectral Coherences based on the CMS and the Fast-SC in a frequency band between $44 \mathrm{kHz}$ and $49 \mathrm{kHz}$. They display alignments of

Table 1

Parameter settings used in the experiments of tested fans.

\begin{tabular}{|c|c|c|c|}
\hline & Case 1 & Case 2 & Case 3 \\
\hline Sampling frequency $F_{s}(\mathrm{kHz})$ & 131.072 & 51.2 & 51.2 \\
\hline Duration $(\mathrm{s})$ & & 5 & \\
\hline$\alpha_{\max }$ in CMS & 1093 & 622 & 909 \\
\hline$N_{w}$ in CMS & 46 & 82 & 144 \\
\hline$N_{w}$ in Fast-SC & & $2^{9}$ & \\
\hline$R$ in Fast-SC & 6 & 12 & 21 \\
\hline Rotation frequency $-f_{\text {rot }}(\mathrm{Hz})$ & 66.6 & 39.9 & 80.1 \\
\hline Ball pass frequency on outer race - BPFO $(\mathrm{Hz})$ & 139.9 & 101.4 & 168.2 \\
\hline Ball pass frequency on inner race - BPFI $(\mathrm{Hz})$ & 259.6 & 177.9 & 312.1 \\
\hline Ball spin frequency - BSF $(\mathrm{Hz})$ & 101.1 & 67.4 & 121.6 \\
\hline Cage frequency - FTF $(\mathrm{Hz})$ & 23.3 & 14.5 & 28.0 \\
\hline
\end{tabular}



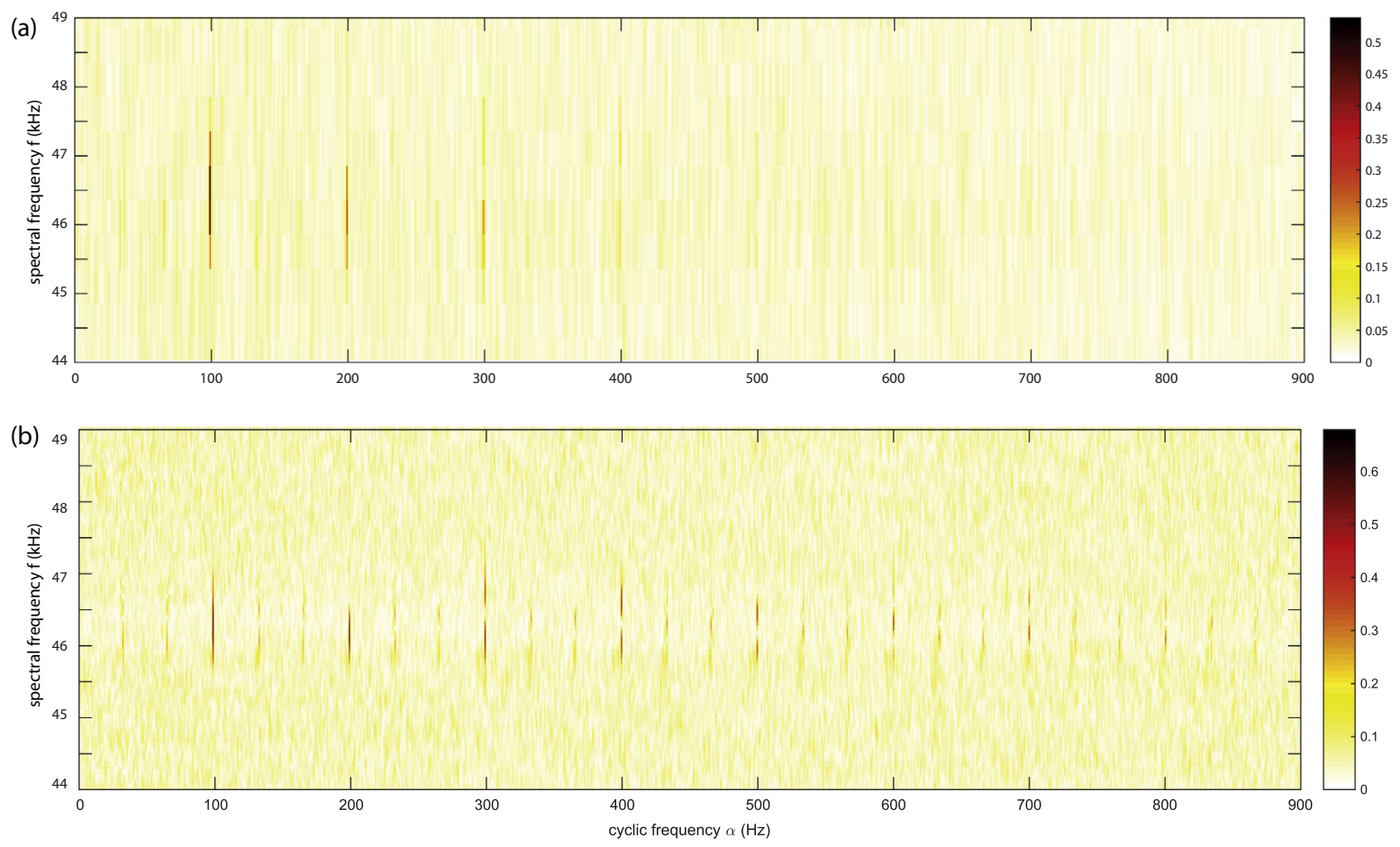

Fig. 14. Case 1. Excerpts of the Spectral Coherence based on (a) the Cyclic Modulation Spectrum $S_{x}^{\text {CMS }}(\alpha, f)(\Delta f=1113 \mathrm{~Hz}, \Delta \alpha=0.2 \mathrm{~Hz})$ and (b) the Fast-SC $S_{x}^{\text {Fast }}(\alpha, f)(\Delta f=100 \mathrm{~Hz}$; Note: the initial cyclic frequency resolution $\Delta \alpha=0.2 \mathrm{~Hz}$ has been decreased to $\Delta \alpha \simeq 2 \mathrm{~Hz}$ after smoothing the images in the horizontally direction in order to match the screen resolution).

non-zero values at cyclic frequencies of the Ball Spin Frequency (BSF) and its multiples up to $900 \mathrm{~Hz}$. It is seen that the frequency resolution allowed by the CMS is not sufficient to reveal some fine details in the Spectral Coherence. In particular, the sidebands due to modulation by the shaft rotation (marked by blue dotted vertical lines) are "erased" in the CMS-based coherence. The same observation is made on the envelope spectra shown in Fig. 15. It is also noteworthy that the Fast-SC-based EES is able to show 9 harmonics of the BSF in the scanned cyclic frequency band, whereas the CMS-based EES can hardly disclose more than 5 harmonics due to the low pass effect described by Eq. (11). A final remark is that the Fast-SC-based EES displays a flat baseline due to the equalization in Eq. (24), which would make easier the design of threshold in statistical tests.

\subsubsection{Case 2}

Excerpts of the Spectral Coherences based on the CMS and the Fast-SC of the second tested fan are displayed in Fig. 16 in the cyclic frequency range $[0 ; 250] \mathrm{Hz}$. They show quite unusual pictures which, at first sight, look more random than structured (i.e. with expected marked vertical lines) despite the variance reduction factor being considerable $(\Delta f / \Delta \alpha=3122$ for the CMS and 500 for the Fast-SC). However, a closer inspection of the Fast-SC in Fig. 16(b) reveals higher "densities of points" along some vertical lines. This is better evidenced, after integration, by the Fast-SC-based EES displayed in Fig. 17(c), which is able to detect the signatures of the outer race (BPFO), the inner race (BPFI), the cage (FTF), and even the rolling elements (BSF) (note that a wider cyclic frequency range than in the excerpts of Fig. 16 is used here). Similar detection is much more difficult with the SES and the CMS-based EES shown in Fig. 17(a) and (b).

Several remarks are in order here. First, the apparent randomness of the CMS and Fast-SC is probably due to a very weak signal produced by the rolling element bearing (this seems to be consolidated by the fact that vertical lines slowly build up when increasing the record length); actually, the bearing might not be faulty at all, but just noisier than expected due to possible mounting imprecision. Second, the CMS-based coherence appears unable to detect this weak cyclostationary signal, probably because it is smeared by a too coarse frequency resolution (as illustrated in Fig. 5(c)).

\subsubsection{Case 3}

This last case resembles very much the previous one, yet with only the signature of the outer race. Note that the BPFO is found significantly lower than the expected value, around $\alpha=160.1 \mathrm{~Hz}$ instead of $168.1 \mathrm{~Hz}$. Here again, both the Spectral Coherences based on the CMS and the Fast-SC (not shown) display a high degree of randomness and only the Fast-SCbased EES can unveil the rolling element bearing signature - see Fig. 18. One possible reason of this superiority is that, 

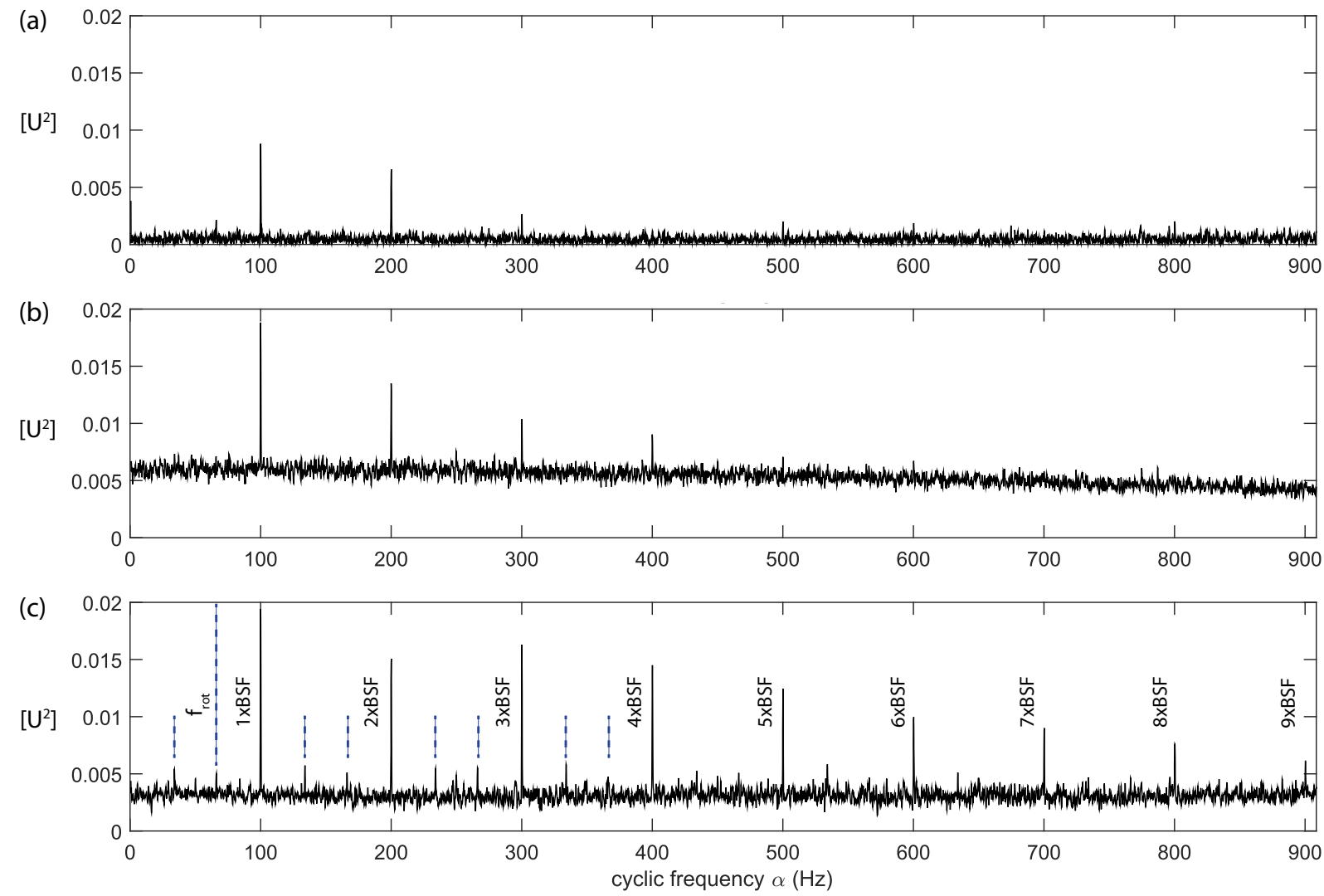

Fig. 15. Case 1. (a) Squared Envelope Spectrum $S_{x}^{S E S}(\alpha)$, (b) CMS-based Enhanced Envelope Spectrum $S_{x}^{E E S}(\alpha)$ and (c) Fast-SC-based Enhanced Envelope Spectrum $S_{x}^{E E S}(\alpha)$ in full band $\left[0 ; F_{s} / 2\right]$ with $\Delta \alpha=0.2 \mathrm{~Hz}$.
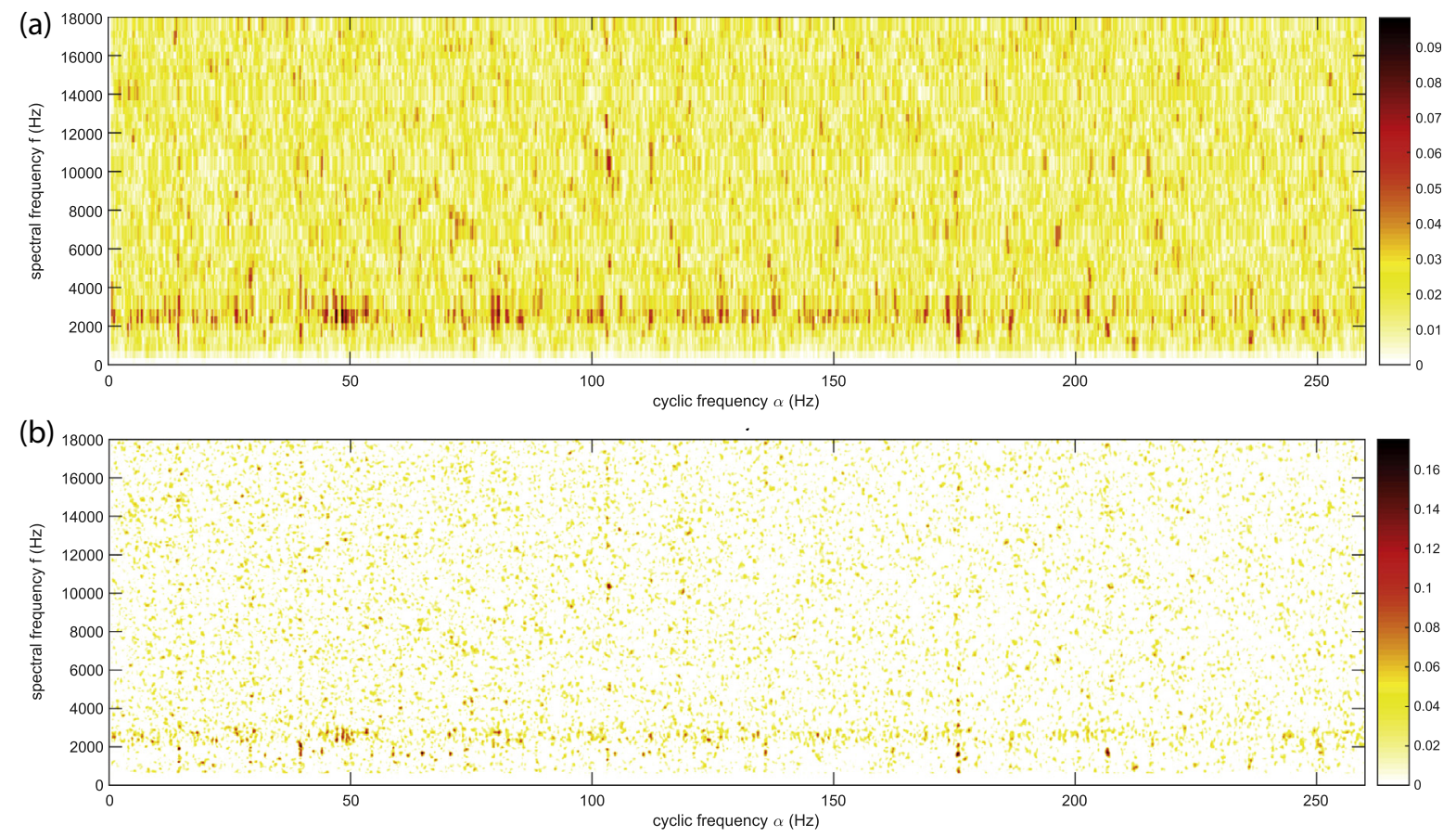

Fig. 16. Case 2. Excerpts of the Spectral Coherence based on (a) the Cyclic Modulation Spectrum $S_{x}^{C M S}(\alpha, f)(\Delta f=624 \mathrm{~Hz}, \Delta \alpha=0.2 \mathrm{~Hz})$ and (b) the Fast-SC $S_{x}^{\text {Fast }}(\alpha, f)(\Delta f=100 \mathrm{~Hz}, \Delta \alpha=0.2 \mathrm{~Hz})$. 

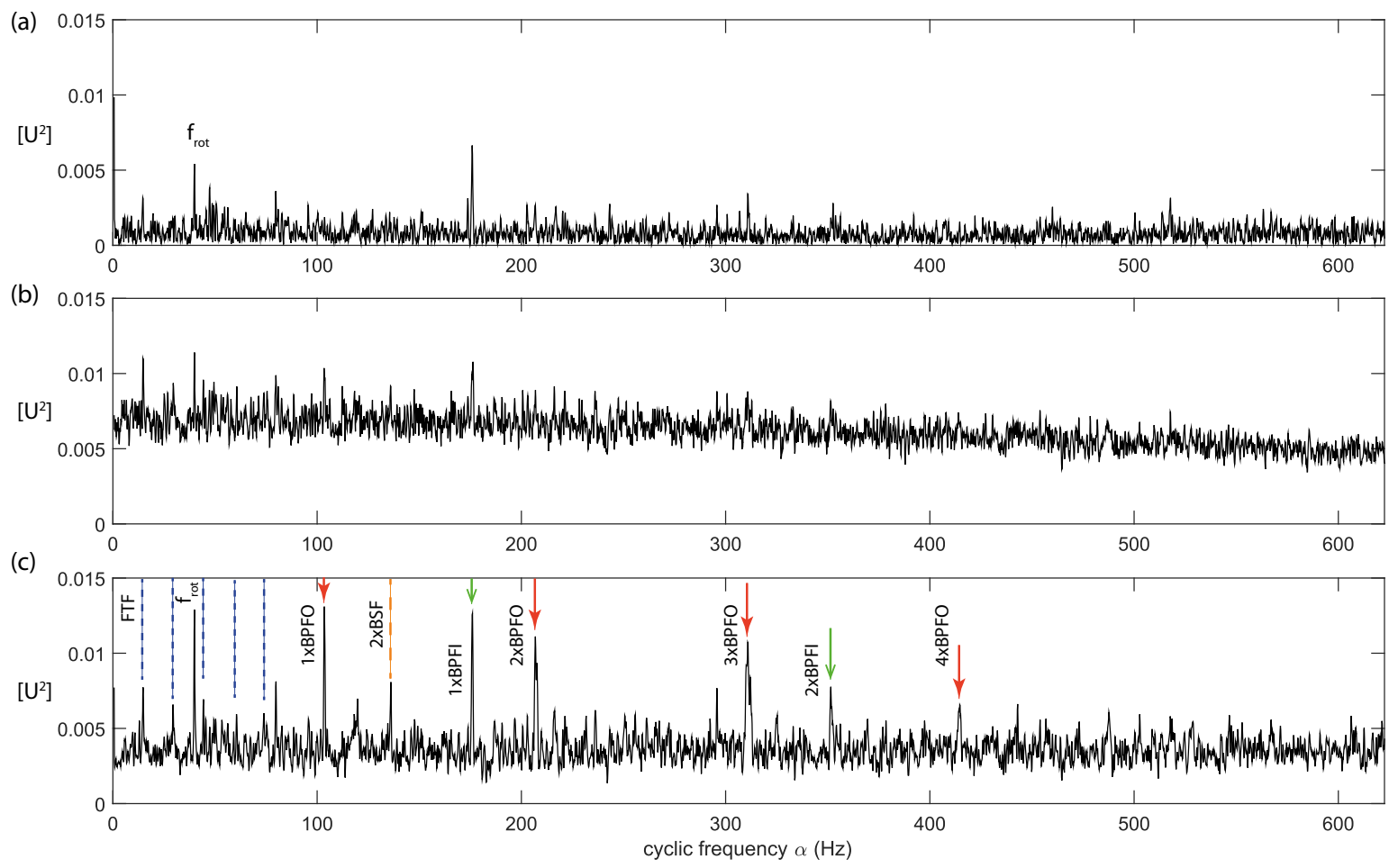

Fig. 17. Case 2. (a) Squared Envelope Spectrum $S_{x}^{S E S}(\alpha)$, (b) CMS-based Enhanced Envelope Spectrum $S_{x}^{\text {EES }}(\alpha)$ and (c) Fast-SC-based Enhanced Envelope Spectrum $S_{x}^{E E S}(\alpha)$ in full band $\left[0 ; F_{s} / 2\right]$ with $\Delta \alpha=0.2 \mathrm{~Hz}$.
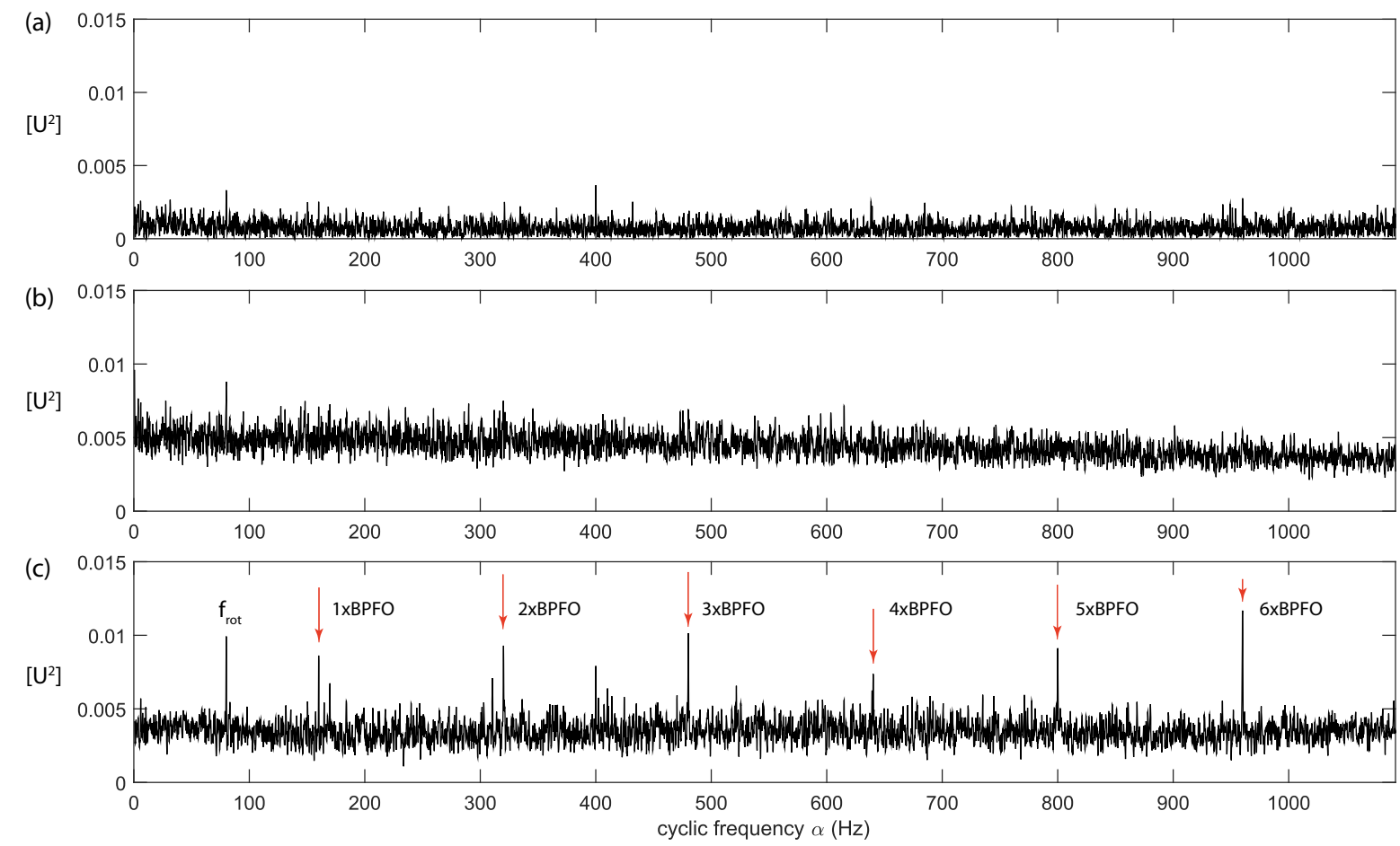

Fig. 18. Case 3. (a) Squared Envelope Spectrum $S_{x}^{\text {SES }}(\alpha)$, (b) CMS-based Enhanced Envelope Spectrum $S_{x}^{E E S}$ and (c) Fast-SC-based Enhanced Envelope Spectrum $S_{x}^{E E S}(\alpha)$ in full band $\left[0 ; F_{s} / 2\right]$ with $\Delta \alpha=0.2 \mathrm{~Hz}$. 
Table 2

Parameter settings used in the experiment of record 277DE.

\begin{tabular}{cc}
\hline Data set & 277DE \\
\hline Sampling frequency $F_{S}(\mathrm{kHz})$ & 12 \\
Duration $(\mathrm{s})$ & 900 \\
$\alpha_{\max }(\mathrm{Hz})$ & 16 \\
$N_{w}$ in CMS & 256 \\
$N_{w}$ in Fast-SC & 9 \\
$R$ in Fast-SC & 28.9 \\
Rotation frequency $f_{\text {rot }}(\mathrm{Hz})$ & 142.9 \\
Ball pass frequency on inner race (fan end) $-\operatorname{BPFI}(\mathrm{Hz})$ & 156.4 \\
Ball pass frequency on inner race (drive end) $-\operatorname{BPFI}(\mathrm{Hz})$ &
\end{tabular}

due to a finer frequency resolution, the numerical evaluation of integral (43) involves more frequency bins for the Fast-SCbased EES than for the CMS-based EES and therefore results in a better averaging of estimation noise.

This situation may be mathematically understood with the help of the following phenomenological model. From inspection of Figs. 16 and 18, the estimated Spectral Coherence seems to behave like

$$
\widehat{\gamma_{x}}(\alpha, f) \sim S_{x}(\alpha) \cdot \epsilon(\alpha, f)
$$

where $\epsilon(\alpha, f)$ is a zero-mean random variable and $S_{x}(\alpha)$ is the theoretical envelope spectrum. According to this model, the complex average

$$
\frac{1}{k_{2}-k_{1}+1}\left|\sum_{k=k_{1}}^{k_{2}} \widehat{\gamma_{x}}\left(\alpha, f_{k}\right)\right| \rightarrow 0
$$

- i.e. converges to zero as $\left(k_{2}-k_{1}+1\right)$ grows - whereas the absolute average

$$
\frac{1}{k_{2}-k_{1}+1} \sum_{k=k_{1}}^{k_{2}}\left|\widehat{\gamma_{x}}\left(\alpha, f_{k}\right)\right| \rightarrow\left|S_{x}(\alpha)\right|
$$

converges to the correct magnitude of the envelope spectrum. Referring back to Eqs. (42) and (43), the SES corresponds to the extreme case represented by the complex average in Eq. (45) and therefore tends to converge destructively as seen in Figs. 17(a) and 18(a), whereas the Fast-SC-based EES corresponds to the extreme case represented by the absolute average in Eq. (46) and therefore tends to converge constructively as seen in Figs. 17(c) and 18(c). The CMS-based EES corresponds to an intermediate case where some of the complex spectral components are first averaged in a large frequency bin of width $\Delta f$ (this is implicit to the use of the STFT ${ }^{1}$ ) before the absolute average is considered; although this intermediate position is clear in Fig. 15(b), the overall effect is rather destructive in Figs. 17(b) and 18(b).

\subsection{Performance evaluation in a benchmark database}

The database of the Case Western Reserve University (CRWU) Bearing Data Center [20] has become a standard benchmark against which newly proposed techniques are often tested. Ref. [21] provides a valuable description of the vibration signals found in the database together with the identification of the difficult cases which are worth consideration when trying to improve upon results obtained from state-of-the-art methods, such as the SES with possible prewhitening [22] and optimal prefiltering with the kurtogram [23]. The Fast-SC and the EES have been systematically computed for all cases investigated in Ref. [21]. The conclusion was that the Fast-SC never performed worse than the reference methods, but could improve the diagnosis in some difficult cases. Only one example is reported here which illustrates quite well the general observation. It relates to record 277DE, a case with an inner-race fault, denoted as "partially successful" for all the 3 methods tested in Ref. [21] (see Table B4 therein). This is a difficult case because the accelerometric sensor is located on the drive-end bearing and is therefore far from the faulty bearing located on the fan-end, on the other side of a large - and possibly noisy - electrical motor.

The parameter settings are given in Table 2 .

Fig. 19(a) and (b) compare excerpts of the Spectral Coherences based on the CMS and the Fast-SC in the band $\left[0 ; F_{s} / 2\right] \mathrm{kHz}$. Although the fundamental of the fan-end inner-race fault is detected in the CMS at $142.3 \mathrm{~Hz}$, it is much less resolved than in the Fast-SC probably because of the coarse frequency resolution. Fig. 19(c) shows a zoomed view of the Fast Spectral

\footnotetext{
${ }^{1}$ Let $X_{L}(f)$ denote the Fourier transform of signal $x\left(t_{n}\right)$ over the full record length $n=0, \ldots, L-1$; then the STFT at time index $i R$ is related to $X_{L}(f)$ as

$$
X_{S T F T}(i, f)=\int_{-F_{s} / 2}^{+F_{s} / 2} X_{L}(f-u) W(u) e^{-j 2 \pi i R u} d u \simeq \int_{-\Delta f / 2}^{\Delta f / 2} X_{L}(f-u) W(u) e^{-j 2 \pi i R u} d u
$$
}

where $W(u)$ stands for the Fourier transform of $w[n]$. 
(a)

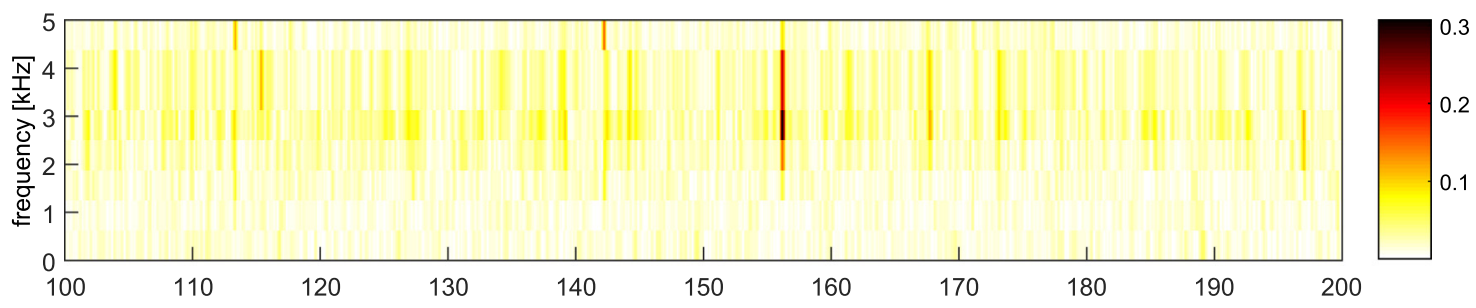

(b)
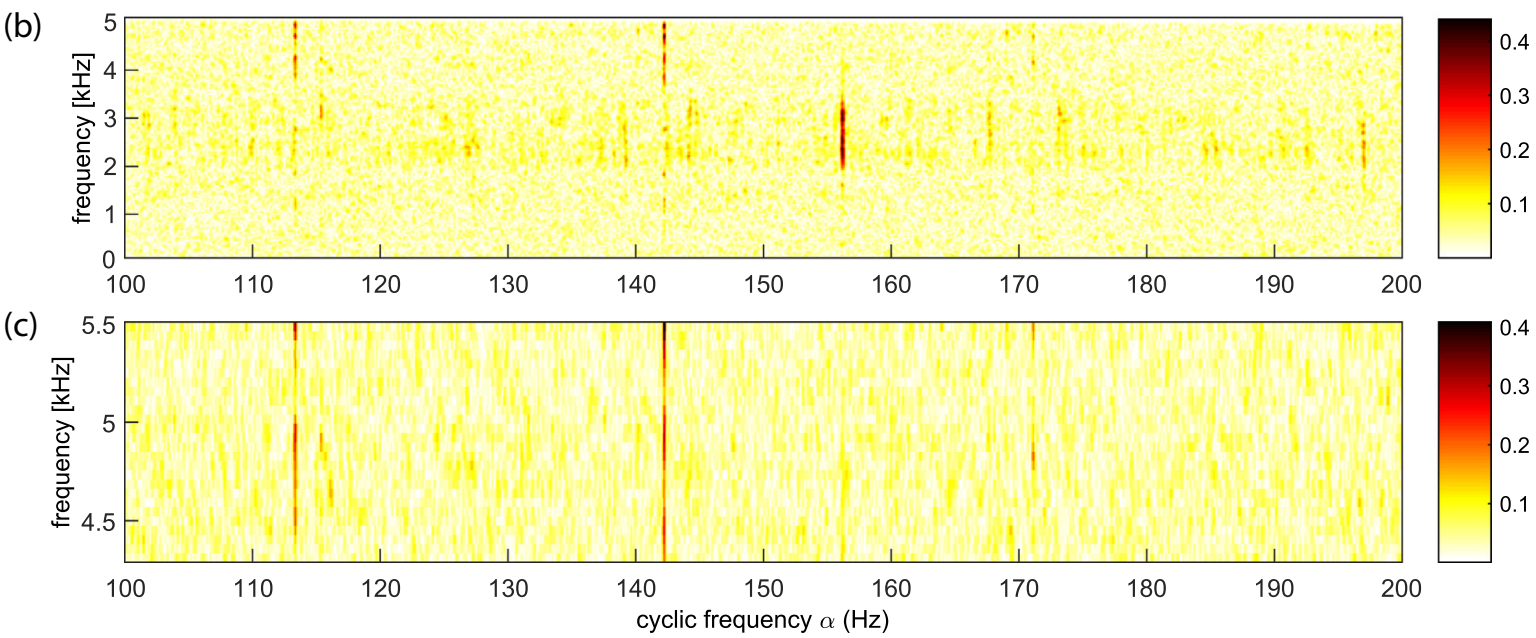

Fig. 19. Excerpts of the Spectral Coherence based on (a) the Cyclic Modulation Spectrum $S_{x}^{\text {CMS }}(\alpha, f)(\Delta f=900 \mathrm{~Hz}, \Delta \alpha=0.1 \mathrm{~Hz})$ and (b) the Fast-SC $S_{x}^{\text {Fast }}(\alpha, f)$ in full band $\left[0 ; F_{s} / 2\right]$ and (c) in selected band $[4.3 ; 5.5] \mathrm{kHz}(\Delta f=50 \mathrm{~Hz}, \Delta \alpha=0.1 \mathrm{~Hz})$.

(a)

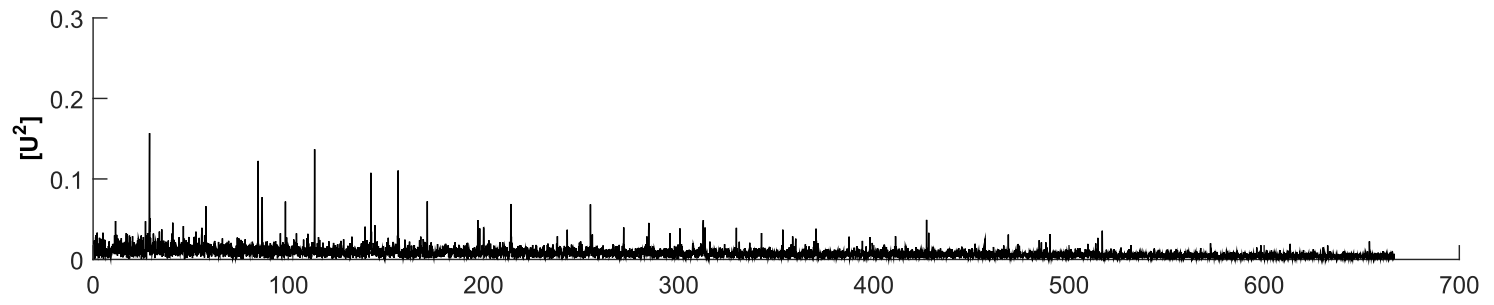

(b)
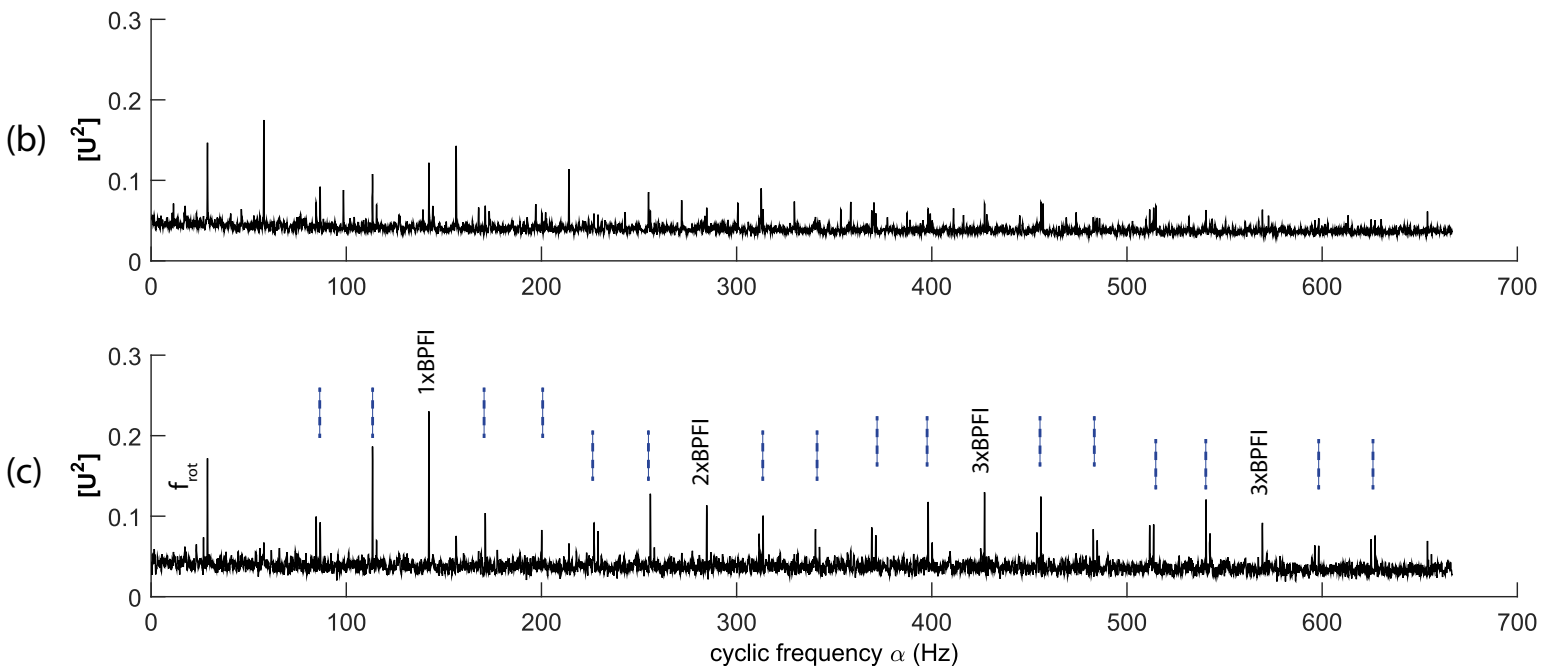

Fig. 20. (a) Squared Envelope Spectrum $S_{x}^{S E S}(\alpha)$, (b) Fast-SC-based Enhanced Envelope Spectrum $S_{x}^{E E S}(\alpha)$ in full band $\left[0 ; F_{S} / 2\right]$ and (c) in selected band $[4.3 ; 5.5] \mathrm{kHz}$ with $\Delta \alpha=0.1 \mathrm{~Hz}$. 


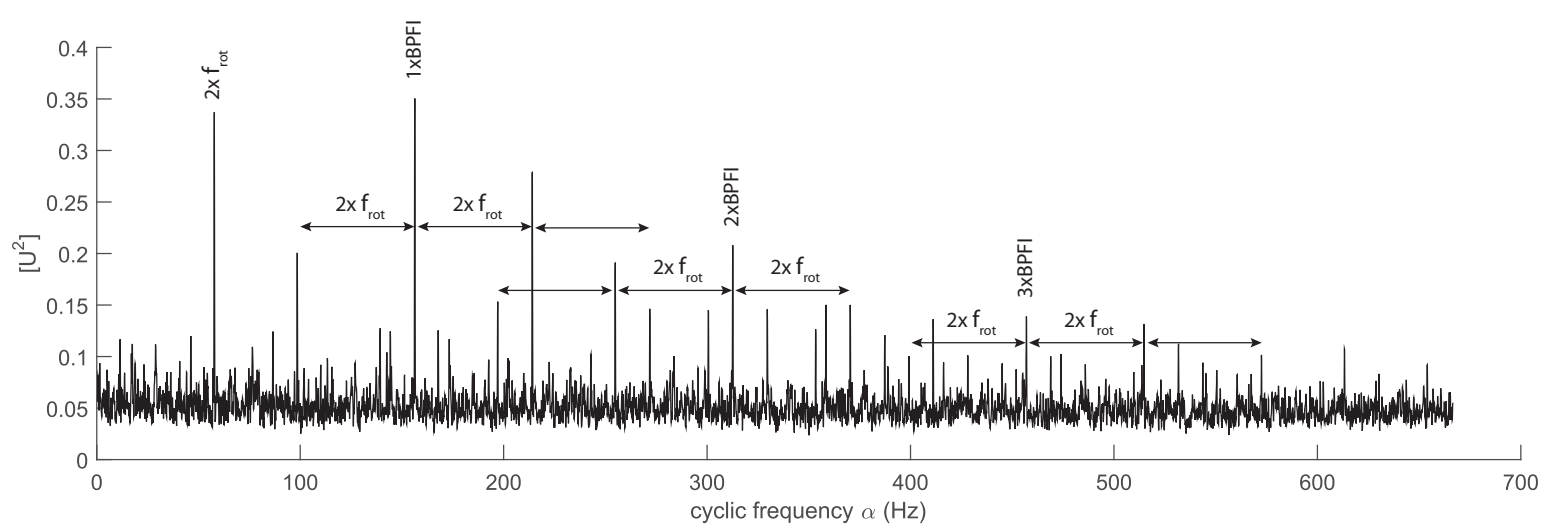

Fig. 21. Fast-SC-based Enhanced Envelope Spectrum $S_{x}^{E E S}(\alpha)$ in selected band $[1.5 ; 3.5] \mathrm{kHz}$ with $\Delta \alpha=0.1 \mathrm{~Hz}$.

Coherence in band $[4.3 ; 5.5] \mathrm{kHz}$ which seems to maximize the SNR. The detection of the fault is further demonstrated by means of the EES computed in this band in Fig. 20. The Fast-SC-based EES clearly evidences the dominant harmonics of the BPFI with sidebands at the shaft rotation, contrary to the classical SES and the CMS-based EES which have a poorer SNR.

It is noted that the fact that the BPFI $\left(=4.947 \times f_{\text {rot }}\right)$ happens to fall close to the fifth multiple of the shaft speed might be troublesome for diagnostics. Here, the very fine cyclic frequency resolution $\Delta \alpha=0.1 \mathrm{~Hz}$ in addition to the detection of higher order harmonics of the fault prevent us from such a confusion.

Incidentally, the Spectral Coherence in Fig. 19(b) also shows a high interference at $\alpha=156.4 \mathrm{~Hz}$ which dominates in the band $[1.5 ; 3.5] \mathrm{kHz}$. The Fast-SC-based EES computed in this band is shown in Fig. 21. It displays the signature of an innerrace fault in the drive-end bearing with marked side-bands at twice the rotation speed. Although no such fault is reported in the literature for the configuration relating to record 277DE, it is believed that the accelerometric sensor (which is close to drive-end bearing) sees a misalignment of the drive-end bearing due to the numerous dismantling operations carried out in the experiment - a loose misaligned inner-race would then have a potential signature at the BPFI with modulations at twice the rotation speed.

\subsection{Diagnostics of bearing under variable regime with the Order-Frequency Fast-SC}

This section illustrates how the proposed Fast-SC can be easily extended to analyze machine signals recorded under varying regime. The consideration of variable operating conditions is not only a necessity in applications where the machine can hardly be operated at constant regime, but it is also apt to provide more diagnostic information than could be obtained otherwise [24,25]. The class of cyclostationary signals has recently been enlarged to account for this situation. Namely, "angle-time" cyclostationarity embodies processes whose statistics are periodic with respect to the angle of rotation of the machine (thus invariant under speed variations) while maintaining a structural description of the carrier that is constant in time [26] (see also Ref. [27] for other generalizations of cyclostationarity). An important statistical quantity for describing angle-time cyclostationary processes is the angle-time autocorrelation function (given here with continuous variables)

$$
R_{x}(\theta, \tau)=\mathbb{E}\left\{x(t(\theta)) x(t(\theta-\tau))^{*}\right\}
$$

which is periodic in $\theta, R_{x}(\theta, \tau)=R_{x}(\theta+\Theta, \tau)$. Its double Fourier transform with respect to $\theta$ and $\tau$ then defines the OrderFrequency Spectral Correlation (OF-SC), $S_{x}(\alpha, f)$, where the cyclic frequency $\alpha$ - now expressed in order - truly reflects the kinematic information of the machine whereas the spectral frequency $f$ - expressed in $\mathrm{Hz}$ - reflects the dynamic information about the propagation medium (e.g. vibration modes). Estimators of the OF-SC have been proposed in Refs. [28-30] based on the ACP. The Fast-SC happens to be very well suited to estimate the OF-SC with the expected gain in computational time discussed in Section 4. The methodology essentially relies on resampling the STFT from the time to the angular domain, while maintaining a constant spectral bandwidth:

1. compute the STFT coefficients $X_{\text {STFT }}(i, f)$ of the signal in the time domain,

2. convert the STFT coefficients to their phase-corrected counterpart $X_{w}(i, f)$ (the Gabor coefficients) using Eq. (3): $X_{w}\left(i, f_{k}\right)=X_{\text {STFT }}\left(i, f_{k}\right) e^{-j 2 \pi t_{i} f_{k}}$ with $t_{i}=i R / F_{s}$,

3. resample $X_{w}\left(i, f_{k}\right)$ from time to angle: $t_{i} \rightarrow \theta_{n}=n \Delta \theta$,

4. convert back to the STFT coefficients: $X_{\text {STFT }}\left(n, f_{k}\right)=X_{w}\left(n, f_{k}\right) e^{j 2 \pi t\left(\theta_{n}\right) f_{k}}$ with $t\left(\theta_{n}\right)$ the time instant corresponding to angle $\theta_{n}$,

5. apply the Fast-SC to $X_{\text {STFT }}\left(n, f_{k}\right)$.

The reason of resampling the Gabor coefficients rather than the STFT coefficients is because the former are much smoother in time than the latter (e.g. see Fig. 7), which follows the idea elaborated in Ref. [31]. 
Table 3

Bearing fault characteristics (orders).

$\begin{array}{ll}\text { Ball pass order on the outer race (BPOO) } & 3.592 \\ \text { Ball pass order on the inner race (BPOI) } & 5.409 \\ \text { Ball spin order (BSO) } & 2.376 \\ \text { Fundamental train (cage) order (FTO) } & 0.399\end{array}$

The methodology is now illustrated for the diagnostics of rolling element bearings under varying regime. For the sake of comparison with previously published works, the data provided as supplementary material in Ref. [32] are analyzed. They consist of measurements taken by a laser vibrometer on a small test-rig with three pre-fabricated bearing faults whose fault orders are reported in Table 3. The machine speed is manually varied between $10 \mathrm{~Hz}$ and $20 \mathrm{~Hz}$ and measured with an encoder mounted on the drive shaft. Sampling frequency is $F_{s}=50 \mathrm{kHz}$ and record duration is $21 \mathrm{~s}$ (see Ref. [32] for a full description of the experimental protocol).

All signals were processed with the Fast OF-SC as explained above, using a Hann window of length $N_{w}=2^{11}$ with $90 \%$ overlap. This returned frequency resolutions of $\Delta f=37 \mathrm{~Hz}$ and $\Delta \alpha=0.004$ order. The STFT was resampled from time to angle by using cubic splines interpolation. The computational time of the STFT was about $2 \mathrm{~s}$ and that of the Fast OF-SC about $26 \mathrm{~s}$ including $6 \mathrm{~s}$ for the resampling process. By comparison, Eq. (34) indicates that about $3 \mathrm{~h}$ would have been necessary to obtain the same results with the ACP.

The results are displayed in Figs. 22(a), 23(a) and 24(a) for the outer-race fault, the inner-race fault, and the ball fault respectively. The Fast-OF coherence clearly evidences the presence of angle-time cyclostationarity with several vertical lines at orders that correspond to the shaft rotation, the bearing faults, their harmonics, and sidebands due to other modulations. They are mainly located in a frequency range below $5 \mathrm{kHz}$, which most probably corresponds to the achievable passband returned by the laser velocity measurements. Close-ups of the Fast-OF coherence show that the bearing faults are correctly identified at the expected orders (see Table 3), except for the ball-fault which has a signature at the cage order (a more typical signature would have been at the ball spin order with sidebands at the cage order). It is also observed that several resonances are excited up to $5 \mathrm{kHz}$ which differ with the type of fault. Note that the Fast-OF coherence preserved a very fine resolution in both the spectral frequency and the order domains, in spite of the speed fluctuations.

The EES was next computed by integrating the OF coherence according to formula (43) in a band below $5 \mathrm{kHz}$ with maximal signal-to-noise ratio and further multiplied with the complementary gain of a sharp combfilter in order to remove harmonics of the shaft orders. Results are displayed in Figs. 22(b), 23(b) and 24(b). They demonstrate typical signatures of the bearing faults with few ambiguity for diagnostics. The outer-race fault shows distinctly the BPOO (ball-pass order on the outer race) and its harmonics with marked sidebands at twice the shaft rotation which might be due to a slight ovalization

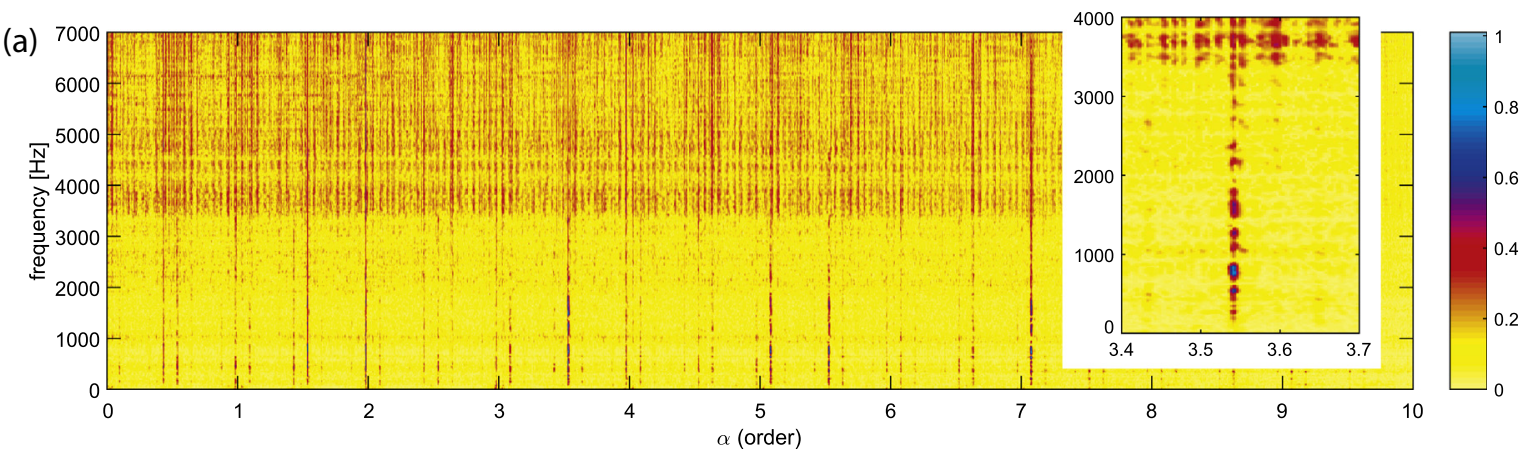

(b)

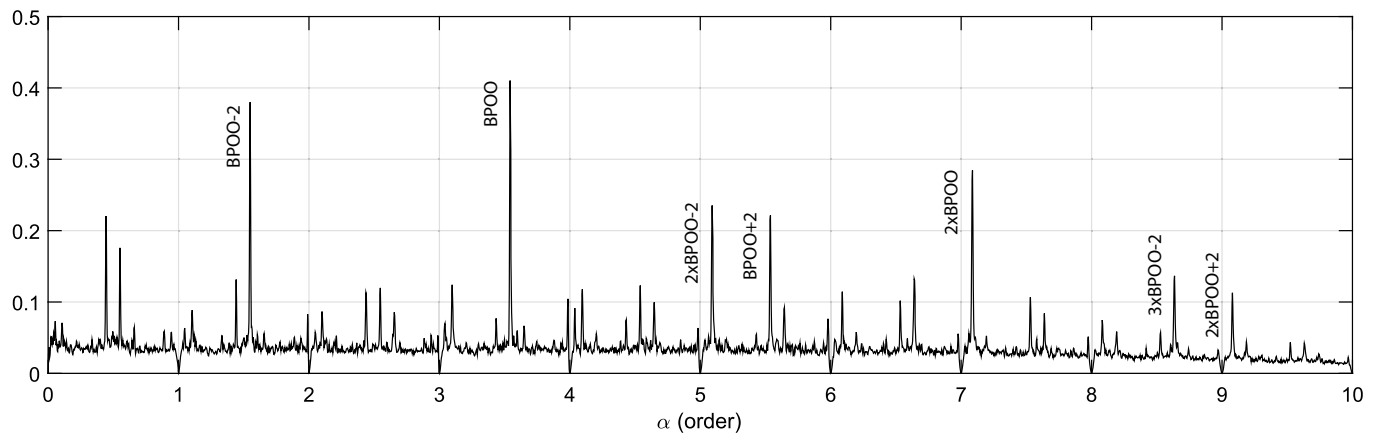

Fig. 22. (a) Spectral Coherence $\gamma_{x}^{\text {Fast }}(\alpha, f)(\Delta f=37 \mathrm{~Hz}, \Delta \alpha=0.004$ order $)$ and Enhanced Envelope Spectrum $S_{x}^{E E S}(\alpha)$ in the band $[0 ; 3]$ kHz. Outer-race fault found at order 3.543 . 


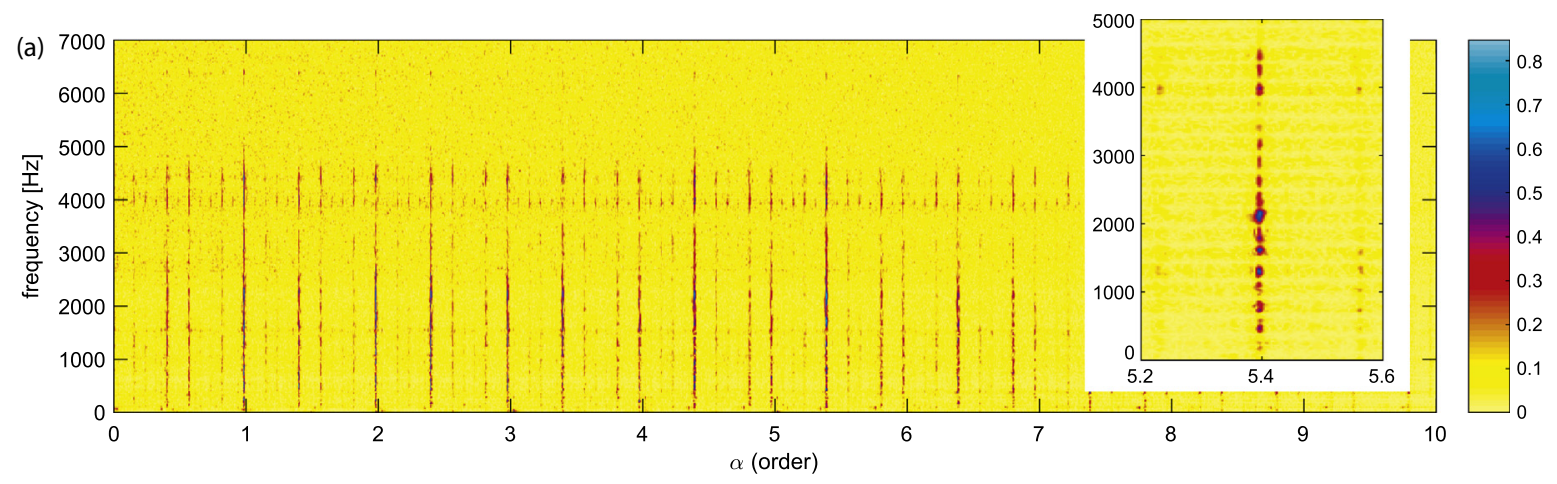

(b) 0.35

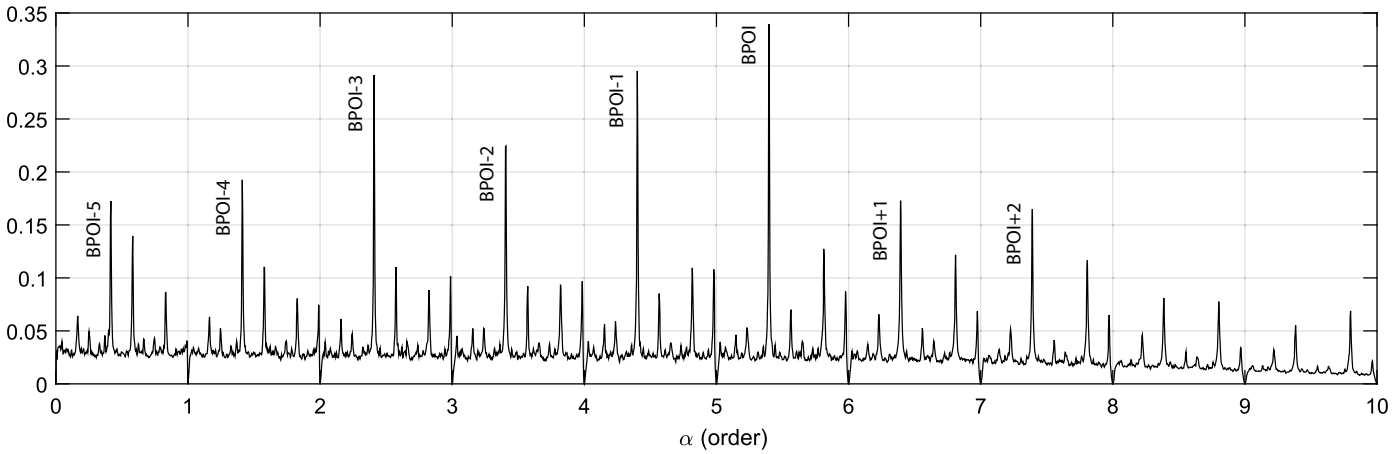

Fig. 23. (a) Spectral Coherence $\gamma_{x}^{\text {Fast }}(\alpha, f)(\Delta f=37 \mathrm{~Hz}, \Delta \alpha=0.004$ order $)$ and Enhanced Envelope Spectrum $S_{x}^{E E S}(\alpha)$ in the band $[0 ; 5]$ kHz. Inner-race fault found at order 5.398 .

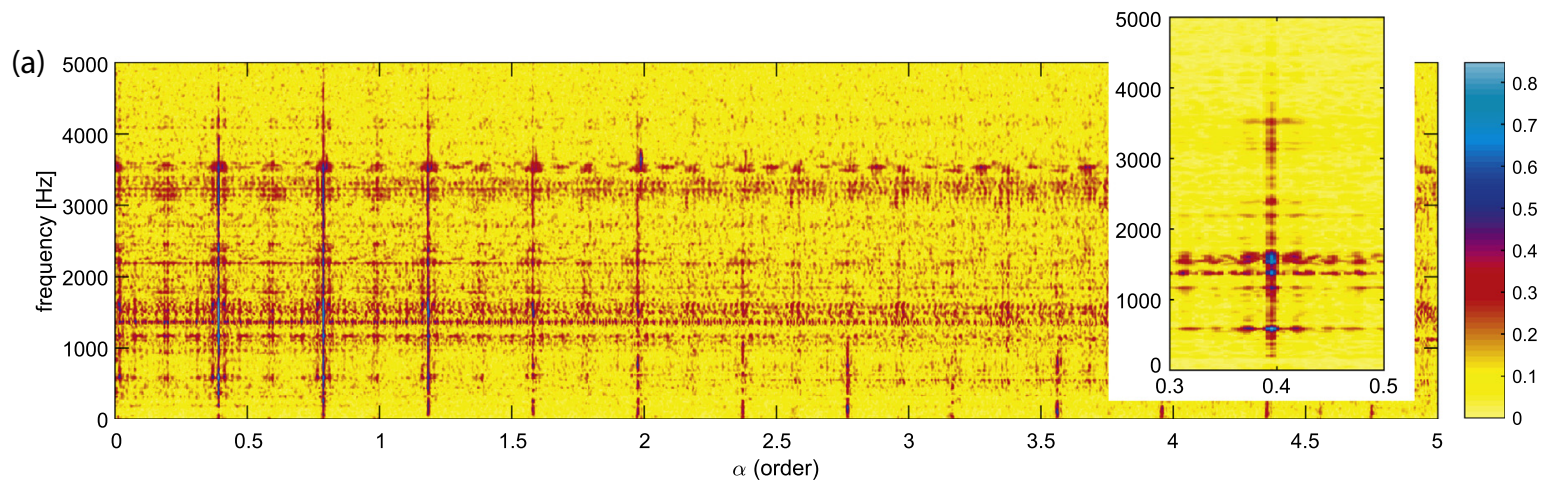

(b)

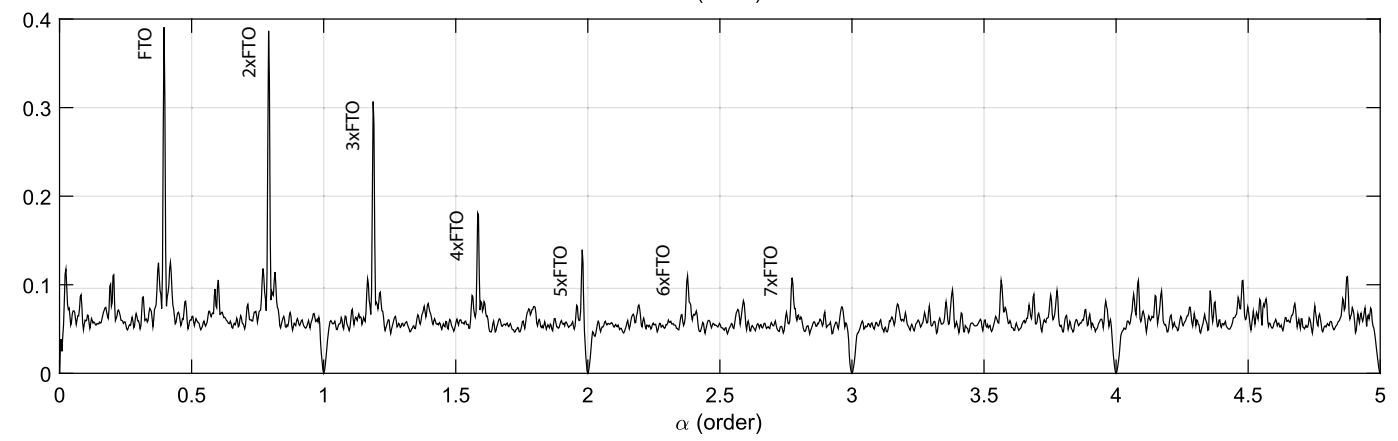

Fig. 24. (a) Spectral Coherence $\gamma_{x}^{\text {Fast }}(\alpha, f)\left(\Delta f=37 \mathrm{~Hz}, \Delta \alpha=0.004\right.$ order) and Enhanced Envelope Spectrum $S_{x}^{E E S}(\alpha)$ in the band $[0 ; 5]$ kHz. Ball fault found at order 0.396. 
of the cage. The inner-race fault shows distinctly the BPOI (ball-pass order on the inner race) with several sidebands at the shaft speed typically due to rotation of the fault in a static but non-homogeneous load distribution. As previously mentioned, the BSO (ball spin order) is missing in the ball fault signature, yet the presence of several harmonics of the cage order is symptomatic of a fault related to it.

By comparison, the approach followed in Ref. [32] was to first to resample the signals in the angular domain, then to denoise them with orthogonal wavelets and to perform a short-term running synchronous average. The processed signal were then bandpass filtered and enveloped in a target band corresponding to $1800-2400 \mathrm{~Hz}$. As mentioned in Ref. [32], this posed a problem since the bandpass filter could not be hold constant in Hertz when performed in the angular domain. Another mentioned problem is that angular resampling "compressed or elongated" the impulse response of the bearing signal so that "too much variation in speed could not be considered".

It is emphasized that no such limitations arise here with the OF-SC. First, no pre-processing is necessary and the signals are analyzed in their entirety. Second, the frequency decomposition of the STFT is applied in the time domain, thus without distortions as would happen if done in the angular domain. Finally, the OF-SC and the corresponding Enhanced Envelope Order Spectrum clearly evidence the fault signature with maximum peaks at the expected fault orders (Ref. [32] introduced further post-processing because the maximum peak in the envelope order spectrum of the inner-race fault did not coincide with the fault order).

\section{Conclusions}

This paper has introduced a new algorithm to compute the Spectral Correlation. Compared to the classical approach based on the Averaged Cyclic Periodogram (ACP), the proposed estimator - coined Fast Spectral Correlation (Fast-SC) - offers a substantial computational gain which makes it very practical when it comes to analyse long records over a wide cyclic frequency range. The gain may be explained by an analogy with the computation of an auto/cross correlation function by means of the FFT algorithm instead of a loop on time-lags. Besides, the Fast-SC is easily amenable to parallel implementation which would still allow further speedup. The Fast-SC has been shown to have similar statistical performance as the ACP, contrary to other fast estimators which are either biased (the Cyclic Modulation Spectrum) or have a non-uniformly bounded variance (the FFT Accumulation Method).

At the same time, the principle of the Fast-SC provides a new interpretation of (second-order) cyclostationarity in terms of periodic correlations between frequency bins of the Short-Time Fourier Transform. The use of the Fast-SC has been illustrated on several vibration signals in order to detect rolling bearing signatures and faults. Obviously, the same procedure can be followed in other domains of applications. The increased computational efficiency of the Fast-SC has also led to the proposal of an Enhanced Envelope Spectrum - an improved version of the envelope spectrum based on the integration of the magnitude of the Fast Spectral Coherence over frequencies. Eventually, it has been shown how the Fast-SC can be easily extended to angle-time cyclostationary signals, for instance when analysing vibration signals of rotating machines captured under nonstationary regimes.

\section{Acknowledgments}

This work was performed within the framework of the Labex CeLyA of University of Lyon, operated by the French National Research Agency.

\section{Appendix A. Proof of Eqs. (11) and (20)}

Let us start by expressing the STFT

$$
X_{\text {STFT }}\left(i, f_{k}\right)=\sum_{m \in \mathbb{Z}} x[i R+m] w[m] e^{-j 2 \pi m_{F_{S}}^{f_{k}}}
$$

(where the summation is now over an infinite number of samples) by means of the spectral decomposition of a discrete stochastic process [33], $x[n]=\int d X(v) e^{j 2 \pi n v / F_{s}}$ and of the data window, $w[n]=\int W(v) e^{j 2 \pi\left(n-N_{0}\right) v / F_{s}} d v$. Inserting the former formulas into the expression of the STFT, one has

$$
X_{S T F T}(i, f)=\iint d X(v) W\left(v^{\prime}\right) \sum_{m \in \mathbb{Z}} e^{j 2 \pi(i R+m) \frac{v}{F_{s}}} e^{j 2 \pi\left(m-N_{0}\right) \frac{v_{F_{s}^{\prime}}}{F}} e^{-j 2 \pi m \frac{f}{F_{S}}} d v^{\prime} .
$$

Let us now evaluate the expected value $C_{i}\left(f_{1}, f_{2}\right)=\mathbb{E}\left\{X_{\text {STFT }}\left(i, f_{2}\right) X_{\text {STFT }}\left(i, f_{1}\right)^{*}\right\}$ :

$$
C_{i}\left(f_{1}, f_{2}\right)=\iiint \int \mathbb{E}\left\{d X\left(v_{2}\right) d X\left(v_{1}\right)^{*}\right\} W\left(v_{2}^{\prime}\right) W\left(v_{1}^{\prime}\right)^{*} \times \sum_{m, m^{\prime} \in \mathbb{Z}^{2}} e^{j 2 \pi(i R+m) \frac{v_{2}}{F_{s}}} e^{-j 2 \pi\left(i R+m^{\prime}\right) \frac{v_{1}}{F_{S}}} e^{j 2 \pi\left(m-N_{0}\right) \frac{v^{\prime}}{F_{s}}} e^{-j 2 \pi\left(m^{\prime}-N_{0}\right) \frac{v_{1}^{\prime}}{F_{s}}} e^{-j 2 \pi m \frac{f_{2}}{F_{S}}} e^{j 2 \pi m^{\prime} \frac{f_{1}}{F_{s}}} d v_{2}^{\prime} d v_{1}^{\prime} .
$$


The above equation involves the quantity $\mathbb{E}\left\{d X\left(v_{2}\right) d X\left(v_{1}\right)^{*}\right\}$ which, for a cyclostationary stochastic process with cyclic frequencies $\left\{\alpha_{k}\right\}_{k \in \mathbb{K}}$, is equal to $\sum_{k \in \mathbb{K}} S_{x}^{k}\left(v_{2}\right) \delta\left(v_{2}-v_{1}-\alpha_{k}\right) d v_{2} d v_{1}$ [4]. Therefore, using the property of the Dirac,

$$
C_{i}\left(f_{1}, f_{2}\right)=\iiint \sum_{k} S_{x}^{k}\left(v_{1}+\alpha_{k}\right) W\left(v_{2}^{\prime}\right) W\left(v_{1}^{\prime}\right)^{*} \times \sum_{m, m^{\prime} \in \mathbb{Z}^{2}} e^{j 2 \pi(i R+m) \frac{\left(v_{1}+\alpha_{k}\right)}{F_{s}}} e^{-j 2 \pi\left(i R+m^{\prime}\right)} \frac{v_{1}}{F_{s}} e^{j 2 \pi\left(m-N_{0}\right) \frac{v_{2}^{\prime}}{F_{s}}} e^{-j 2 \pi\left(m^{\prime}-N_{0}\right) \frac{v_{1}^{\prime}}{F_{s}}} e^{-j 2 \pi m \frac{f_{2}}{F_{s}}} e^{j 2 \pi m} \frac{f_{1}}{F_{s}} d v_{2}^{\prime} d v_{1}^{\prime} d v_{1} .
$$

The next step is to recognize that $\sum_{m \in \mathbb{Z}} e^{j 2 \pi m v / F_{s}}=F_{s} \delta(v)$ for $|v|<F_{s} / 2$. Therefore,

$$
C_{i}\left(f_{1}, f_{2}\right)=F_{s} \int \sum_{k} S_{x}^{k}\left(v_{1}+\alpha_{k}\right) W\left(f_{2}-v_{1}-\alpha_{k}\right) W\left(f_{1}-v_{1}\right)^{*} d v_{1} e^{j 2 \pi\left(i R+N_{0}\right) \frac{\alpha_{k}}{F_{s}}} e^{-j 2 \pi N_{0} \frac{\left(f_{2}-f_{1}\right)}{F_{s}}} .
$$

Now, if $S_{x}^{k}(f)$ is assumed smooth enough in $f$ to be almost constant as compared to $W(f)$,

$$
\begin{aligned}
& \int S_{x}^{k}\left(v_{1}+\alpha_{k}\right) W\left(f_{2}-v_{1}-\alpha_{k}\right) W\left(f_{1}-v_{1}\right)^{*} d v_{1} \\
& \simeq S_{x}^{k}\left(f_{2}\right) \int W\left(f_{2}-v_{1}-\alpha_{k}\right) W\left(f_{1}-v_{1}\right)^{*} d v_{1} \\
& =S_{x}^{k}\left(f_{2}\right) R_{w}\left(\alpha_{k}-f_{2}+f_{1}\right)
\end{aligned}
$$

with $R_{w}(f)$ (an even function) as defined in Eq. (12). Finally, setting $f_{2}=f$ and $f_{1}=f-p \Delta f$ in $C_{i}\left(f_{1}, f_{2}\right)$, one arrives at

$$
\mathbb{E}\left\{X_{S T F T}(i, f) X_{S T F T}(i, f-p \Delta f)^{*}\right\} \simeq e^{-j 2 \pi N_{0} p \frac{\Delta f}{F_{S}} F_{S}} \sum_{k} \underbrace{S_{x}^{k}(f) R_{w}\left(\alpha_{k}-p \Delta f\right)}_{c_{k}} e^{j 2 \pi\left(i R+N_{0}\right) \frac{\alpha_{k}}{\frac{\alpha_{F}}{F_{S}}}}
$$

The latter expression shows that $e^{j 2 \pi N_{0} p \frac{\Delta f}{F_{s}}} F_{s}^{-1} \mathbb{E}\left\{X_{\text {STFT }}(i, f) X_{\text {STFT }}(i, f-p \Delta f)^{*}\right\}$ has a Fourier series with Fourier coefficients $c_{k}=S_{x}^{k}(f) R_{w}\left(\alpha_{k}-p \Delta f\right)$. Therefore,

$$
\frac{1}{K F_{S}} \sum_{i=0}^{K-1} \mathbb{E}\left\{X_{S T F T}(i, f) X_{S T F T}(i, f-p \Delta f)^{*}\right\} e^{-j 2 \pi\left(i R+N_{0}\right)_{\frac{\alpha_{k}}{F_{s}}}} e^{j 2 \pi N_{0} p_{F_{S}} \frac{\Delta f}{F_{S}}}
$$

is an evaluation of $c_{k}$. The latter expression is recognized as the second line of Eq. (19), thus proving result (20). Result (11) comes as a particular case when $p=0$.

\section{Appendix B. Frequency resolution}

The frequency resolution of the Fast-SC is evaluated by comparing its expression to the theoretical SC when the signal of interest is made of a pure complex exponential, $x\left(t_{n}\right)=e^{j 2 \pi \alpha_{0} t_{n}}$. In this case, the SC is actually made of a product of a continuous Dirac and a discrete Dirac,

$$
S_{x}(\alpha, f)=F_{s}^{-1} \delta\left(f-\alpha_{0}\right) \delta[\alpha],
$$

(where $\delta[\alpha]=1$ if $\alpha=0$ and 0 otherwise) as found from direct application of definition (6).

From Eq. (24), the expected value of the Fast-SC then reads

$$
\mathbb{E}\left\{S_{x}^{\mathrm{Fast}}(\alpha, f)\right\}=\frac{\sum_{p=0}^{P} \mathbb{E}\left\{S_{x}(\alpha, f ; p)\right\}}{\sum_{p=0}^{P} R_{w}(\alpha-p \Delta f)} R_{w}(0),
$$

where the quantity

$$
\mathbb{E}\left\{S_{X}(\alpha, f ; p)\right\}=\frac{1}{K\|w\|^{2} F_{S}} \sum_{i=0}^{K-1} \mathbb{E}\left\{X_{S T F T}(i, f) X_{S T F T}(i, f-p \Delta f)^{*}\right\} e^{-j 2 \pi \frac{\alpha}{F_{S}}\left(i R+N_{0}\right)} e^{j 2 \pi \frac{p N_{0}}{N_{W}}}
$$

appearing in the above equation has been addressed in Eq. Appendix A. In the case of a pure complex exponential, it comes $d X(v)=\delta\left(v-\alpha_{0}\right) d v$ and therefore $\mathbb{E}\left\{d X\left(v_{2}\right) d X\left(v_{1}\right)^{*}\right\}=\delta\left(v_{2}-\alpha_{0}\right) \delta\left(v_{1}-\alpha_{0}\right) d v_{2} d v_{1}$ in Eq. (A.3). Hence,

$$
\begin{aligned}
\mathbb{E}\left\{S_{x}(\alpha, f ; p)\right\} & =\frac{1}{K\|w\|^{2} F_{S}} W\left(f-\alpha_{0}\right) W\left(f-\alpha_{0}-p \Delta f\right)^{*} \sum_{i=0}^{K-1} e^{-j 2 \pi \frac{\alpha}{F_{s}}\left(i R+N_{0}\right)} \\
& =\frac{1}{K\|w\|^{2} F_{S}} W\left(f-\alpha_{0}\right) W\left(f-\alpha_{0}-p \Delta f\right)^{*} D_{K}\left(\frac{\alpha}{F_{S}}\right) e^{-j 2 \pi \tau_{F_{S}}\left(N_{0}+R(K-1) / 2\right)}
\end{aligned}
$$


where $D_{K}(x)=\sin (\pi K x) / \sin (\pi x)$ stands for the Dirichlet kernel. Eq. (B.2) then becomes

$$
\mathbb{E}\left\{S_{x}^{\text {Fast }}(\alpha, f)\right\}=W\left(f-\alpha_{0}\right) D_{K}\left(\frac{\alpha}{F_{s}}\right) \frac{\sum_{p=0}^{P} W\left(f-\alpha_{0}-p \Delta f\right)^{*}}{\sum_{p=0}^{P} R_{w}(\alpha-p \Delta f)} \frac{R_{w}(0)}{K\|w\|^{2} F_{S}} e^{-j 2 \pi_{F_{s}}\left(N_{0}+R(K-1) / 2\right)} .
$$

Now, using the facts that $R_{w}(0)=\|w\|^{2}, \sum_{p=0}^{P} W\left(f-v_{0}+\alpha_{k}-p \Delta f\right)=w\left[N_{0}\right] N_{w} / F_{s}$ and $\sum_{p=0}^{P} R_{w}(\alpha-p \Delta f)=\left|w\left[N_{0}\right]\right|^{2} N_{w} / F_{s}$ in the frequency range of interest, it finally comes

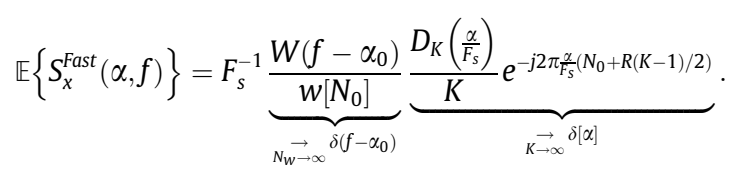

Comparison of Eq. (B.7) to the theoretical SC in Eq. (B.1) shows that

1. the frequency resolution $\Delta f$ is governed by the bandwidth of $W(f)$, that is $\Delta f \sim F_{s} / N_{w}$,

2. the cyclic frequency resolution $\Delta \alpha$ is governed by the bandwidth of $D_{K}\left(\alpha / F_{s}\right)$, that is $\Delta \alpha \sim F_{s} / L$.

These results are similar to those of the classical estimators of the SC, for instance as discussed in Ref. [1]. They also prove that the frequency resolution of the Fast-SC is independent of frequency $f$, contrary to the computationally efficient estimator introduced in Ref. [18].

\section{Appendix C. Variance of the FastSC}

The variance of the Fast-SC is calculated here for $R=1$. This choice not only returns the minimum achievable variance which is closely approached by the high fraction of overlap advocated in Section 5 , but is also significantly simplifies the calculations. The variance reads

$$
\operatorname{Var}\left\{S_{x}^{\text {Fast }}(\alpha, f)\right\}=\left|\frac{R_{w}(0)}{\sum_{p=0}^{P} R_{w}(\alpha-p \Delta f)}\right|^{2} \operatorname{Var}\left\{\sum_{p=0}^{P} S_{x}(\alpha, f ; p)\right\}
$$

where

$$
\operatorname{Var}\left\{\sum_{p=0}^{P} S_{x}(\alpha, f ; p)\right\}=\sum_{p, p^{\prime}} \mathbb{E}\left\{S_{x}(\alpha, f ; p) S_{x}\left(\alpha, f ; p^{\prime}\right)^{*}\right\}-\mathbb{E}\left\{S_{x}(\alpha, f ; p)\right\} \mathbb{E}\left\{S_{x}\left(\alpha, f ; p^{\prime}\right)\right\}^{*} .
$$

Substituting $S_{x}(\alpha, f ; p)$ for its expression in Eq. (19) and assuming that the STFT coefficients are Gaussian distributed, ${ }^{2}$ it is found that ${ }^{3}$

$$
\begin{aligned}
\operatorname{Var}\left\{\sum_{p=0}^{P} S_{X}(\alpha, f ; p)\right\}= & \kappa^{2} \sum_{i, i^{\prime}} \sum_{p, p^{\prime}}\left(\mathbb{E}\left\{X_{\text {STFT }}(i, f) X_{\text {STFT }}\left(i^{\prime}, f\right)^{*}\right\} \mathbb{E}\left\{X_{\text {STFT }}(i, f-p \Delta f)^{*} X_{S T F T}\left(i^{\prime}, f-p^{\prime} \Delta f\right)\right\}\right. \\
& \left.+\mathbb{E}\left\{X_{\text {STFT }}(i, f) X_{\text {STFT }}\left(i^{\prime}, f-p^{\prime} \Delta f\right)\right\} \mathbb{E}\left\{X_{\text {STFT }}(i, f-p \Delta f)^{*} X_{\text {STFT }}\left(i^{\prime}, f\right)^{*}\right\}\right) \times e^{j 2 \pi\left(i-i^{\prime}\right) R_{F_{s}}^{\alpha}} e^{j 2 \pi\left(p-p^{\prime}\right) N_{0}} N_{N_{w}}
\end{aligned}
$$

where $\kappa^{2}=\left(K\|w\|^{2} F_{s}\right)^{-2}$. The above expression contains the general term $C_{i, i^{\prime}}\left(f_{1}, f_{2}\right)=\mathbb{E}\left\{X_{\text {STFT }}\left(i, f_{2}\right) X_{\text {STFT }}\left(i^{\prime}, f_{1}\right)^{*}\right\}$ (it is recalled that $\left.X_{\text {STFT }}(i, f)=X_{\text {STFT }}(i,-f)^{*}\right)$. Following similar lines as in Appendix A, it can be shown that

$$
C_{i, i^{\prime}}\left(f_{1}, f_{2}\right)=\sum_{k} e^{-j 2 \pi N_{0} \frac{\left(f_{2}-f_{1}-\alpha_{k}\right)}{F_{s}}} e^{j 2 \pi i i_{F_{s}}} \int S_{x}^{k}\left(v+\alpha_{k}\right) W\left(f_{2}-v-\alpha_{k}\right) W\left(f_{1}-v\right)^{*} e^{j 2 \pi\left(i-i^{\prime}\right) R_{F_{s}}^{v}} d v
$$

Therefore,

$$
\begin{aligned}
\operatorname{Var}\left\{\sum_{p=0}^{P} S_{x}(\alpha, f ; p)\right\}= & \kappa^{2} \sum_{p, p^{\prime}} \sum_{i, i^{\prime}} \sum_{k, k^{\prime}} e^{j 2 \pi\left(i+N_{0}\right) \frac{\left(\alpha_{k}-\alpha_{k^{\prime}}\right)}{F_{s}}} e^{j 2 \pi \pi_{N_{w}}^{N_{0}}\left(p^{\prime}-p\right)} \iint S_{x}^{k}\left(v+\alpha_{k}\right) S_{x}^{k^{\prime}}\left(v^{\prime}+\alpha_{k^{\prime}}\right)^{*} \\
& \times\left(W\left(f-v-\alpha_{k}\right) W(f-v)^{*} W\left(f-p \Delta f-v^{\prime}-\alpha_{k^{\prime}}\right)^{*} W\left(f-p^{\prime} \Delta f-v^{\prime}\right)\right. \\
& \left.+W\left(f-v-\alpha_{k}\right) W\left(-f+p^{\prime} \Delta f-v\right)^{*} W\left(f-p \Delta f-v^{\prime}-\alpha_{k^{\prime}}\right)^{*} W\left(-f-v^{\prime}\right)\right) e^{j 2 \pi\left(i-i^{\prime}\right) \frac{\left(v-v^{\prime}-\alpha\right)}{f_{s}}} d v d v^{\prime} .
\end{aligned}
$$

\footnotetext{
${ }^{2}$ According to the central limit theorem, the STFT coefficient quickly converge to a Gaussian law [33].

${ }^{3}$ If $X_{1}, X_{2}, X_{3}$ and $X_{4}$ are four zero-mean complex Gaussian random variables, then $\mathbb{E}\left\{X_{1} X_{2}^{*} X_{3} X_{4}^{*}\right\}=\mathbb{E}\left\{X_{1} X_{2}^{*}\right\} \mathbb{E}\left\{X_{3} X_{4}^{*}\right\}+\mathbb{E}\left\{X_{1} X_{3}\right\} \mathbb{E}\left\{X_{2}^{*} X_{4}^{*}\right\}+\mathbb{E}\left\{X_{1} X_{4}^{*}\right\} \mathbb{E}\left\{X_{2}^{*} X_{3}\right\}$.
} 

Thus,

Considering first the sum over index $i^{\prime}$, one has $\sum_{i=1}^{K} e^{-j 2 \pi i^{\prime}\left(v-v^{\prime}-\alpha\right) / F_{s}}$ tending towards $F_{s} \delta\left(v-v^{\prime}-\alpha\right)$ for $\left|v-v^{\prime}-\alpha\right|<F_{s} / 2$.

$$
\begin{aligned}
\operatorname{Var}\left\{\sum_{p=0}^{P} S_{x}(\alpha, f ; p)\right\} \simeq & F_{s} \kappa^{2} \sum_{p, p^{\prime}} \sum_{i} \sum_{k, k^{\prime}} e^{j 2 \pi\left(i+N_{0}\right)} \frac{\left.\alpha_{k}-\alpha_{k^{\prime}}\right)}{F_{s}} e^{j 2 \pi_{N_{w}}^{N_{0}}\left(p^{\prime}-p\right)} \int S_{x}^{k}\left(v+\alpha_{k}\right) S_{x}^{k^{\prime}}\left(v-\alpha+\alpha_{k^{\prime}}\right)^{*} \\
& \times\left(W\left(f-v-\alpha_{k}\right) W(f-v)^{*} W\left(f-p \Delta f-v-\alpha-\alpha_{k^{\prime}}\right)^{*} W\left(f-p^{\prime} \Delta f-v-\alpha\right)\right. \\
& \left.+W\left(f-v-\alpha_{k}\right) W\left(-f+p^{\prime} \Delta f-v\right)^{*} W\left(f-p \Delta f-v-\alpha-\alpha_{k^{\prime}}\right)^{*} W(-f-v-\alpha)\right) d v .
\end{aligned}
$$

Next, considering the sum over index $i$ and assuming that the set $\mathbb{K}$ of cyclic frequencies is such that $\alpha_{k}-\alpha_{k^{\prime}} \neq q F_{s} / R, \forall q \in \mathbb{Z}$, one has $K^{-1} \sum_{i=1}^{K} e^{j 2 \pi i R\left(\alpha_{k}-\alpha_{k^{\prime}}\right) / F_{s}}$ tending towards the discrete Dirac $\delta\left[\alpha_{k}-\alpha_{k^{\prime}}\right]$. Hence,

$$
\begin{aligned}
\operatorname{Var}\left\{\sum_{p=0}^{P} S_{x}(\alpha, f ; p)\right\} \simeq & K F_{s} \kappa^{2} \sum_{p, p^{\prime}} \sum_{k} e^{j 2 \pi \pi_{N_{W}}^{N_{0}}\left(p^{\prime}-p\right)} \int S_{x}^{k}\left(v+\alpha_{k}\right) S_{x}^{k}\left(v-\alpha+\alpha_{k}\right)^{*} \\
& \times\left(W\left(f-v-\alpha_{k}\right) W(f-v)^{*} W\left(f-p \Delta f-v-\alpha-\alpha_{k}\right)^{*} W\left(f-p^{\prime} \Delta f-v-\alpha\right)\right. \\
& \left.+W\left(f-v-\alpha_{k}\right) W\left(-f+p^{\prime} \Delta f-v\right)^{*} W\left(f-p \Delta f-v-\alpha-\alpha_{k}\right)^{*} W(-f-v-\alpha)\right) d v .
\end{aligned}
$$

The rest of the proof is to recognize that because kernel $W(f)$ is narrowband - with width equal to $\Delta f$ - the product of its frequency shifted versions in the second line of Eq. (C.7) is close to zero except for $p=p^{\prime}$. Similarly, the product of its frequency shifted versions in the third line of Eq. (C.7) is close to zero almost everywhere (except around a discrete set of frequencies which is hereafter neglected). Eq. (C.7) then becomes

$$
\begin{aligned}
\operatorname{Var}\left\{\sum_{p=0}^{P} S_{x}(\alpha, f ; p)\right\} \simeq & K F_{s} \kappa^{2} \sum_{k} \int S_{x}^{k}\left(v+\alpha_{k}\right) S_{x}^{k}\left(v-\alpha+\alpha_{k}\right)^{*} \\
& \times\left(W\left(f-v-\alpha_{k}\right) W(f-v)^{*} \sum_{p} W\left(f-p \Delta f-v-\alpha-\alpha_{k}\right)^{*} W(f-p \Delta f-v-\alpha)\right) d v .
\end{aligned}
$$

Finally, assuming as in Appendix A that $S_{x}(\alpha, f)$ is smooth enough in $f$ to be almost constant as compared to $W(f)$, recalling that $R_{w}(0)=\|w\|^{2}$ and using the change of variable $u=f-v$, the variance of the Fast-SC reads

$$
\operatorname{Var}\left\{S_{x}^{\text {Fast }}(\alpha, f)\right\} \simeq \frac{1}{K} \sum_{k \in \mathbb{K}} K_{w}\left(\alpha_{k}, \alpha\right) S_{x}^{k}(f) S_{x}^{k}(f-\alpha)^{*}
$$

with

$$
K_{w}\left(\alpha_{k}, \alpha\right)=\frac{\sum_{p=0}^{P} \int W\left(u-\alpha_{k}\right) W(u)^{*} W\left(u-p \Delta f-\alpha-\alpha_{k}\right)^{*} W(u-p \Delta f-\alpha) d u}{F_{s}\left|\sum_{p=0}^{P} R_{w}(\alpha-p \Delta f)\right|^{2}} .
$$

A rough approximation to (Eq. C.10) is eventually obtained by recognizing that $\sum_{p=0}^{P} R_{w}(\alpha-p \Delta f) \approx w\left[N_{0}\right]^{2} N_{w} / F_{s}$, $\sum_{p} W\left(u-p \Delta f-\alpha-\alpha_{k}\right)^{*} W(u-p \Delta f-\alpha) \approx R_{w}\left(\alpha_{k}\right) N_{w} / F_{s}$ (equality would actually hold if the summation was over $p \in \mathbb{Z}$ ) and $\int W\left(u-\alpha_{k}\right) W(u)^{*} d u=R_{w}\left(\alpha_{k}\right)$, leading to

$$
K_{w}\left(\alpha_{k}, \alpha\right) \approx \frac{R_{w}\left(\alpha_{k}\right)^{2}}{N_{w}^{2} w\left[N_{0}\right]^{4}} .
$$

It is noteworthy that for $\alpha_{k}=0, \sqrt{K_{w}(0, \alpha)} \approx \sum_{n} w[n]^{2} /\left(N_{w} w\left[N_{0}\right]^{2}\right)$ is recognized as a measure of the bandwidth of $w[n]$.

\section{References}

[1] J. Antoni, Cyclic spectral analysis in practice, Mech. Syst. Signal Process. 21 (2) (2007) 597-630.

[2] W. Gardner, Measurement of spectral correlation, IEEE Trans. Acoust., Speech, and Signal Process. 34 (5) (1986) 1111-1123.

[3] W.A. Gardner, Exploitation of spectral redundancy in cyclostationary signals, IEEE Signal Process. Mag. 8 (2) (1991) 14-36.

[4] H.L. Hurd, Correlation theory of almost periodically correlated processes, J. Multivariate Anal. 37 (1) (1991) 24-45.

[5] D. Dehay, Spectral analysis of the covariance of the almost periodically correlated processes, Stochast. Process. Appl. 50 (2) (1994) 315-330.

[6] I. Javorskyj, J. Leskow, I. Kravets, I. Isayev, E. Gajecka, Linear filtration methods for statistical analysis of periodically correlated random processes - part i: coherent and component methods and their generalization, Signal Process. 92 (7) (2012) 1559-1566.

[7] C. Capdessus, M. Sidahmed, J. Lacoume, Cyclostationary processes: application in gear faults early diagnosis, Mech. Syst. Signal Process. 14 (3) (2000) $371-385$.

[8] I. Antoniadis, G. Glossiotis, Cyclostationary analysis of rolling-element bearing vibration signals, J. Sound Vib. 248 (5) (2001) 829-845.

[9] R. Randall, J. Antoni, S. Chobsaard, The relationship between spectral correlation and envelope analysis in the diagnostics of bearing faults and other cyclostationary machines signals, Mech. Syst. Signal Process. 15 (5) (2001) 945-962.

[10] J. Antoni, F. Bonnardot, A. Raad, M.E. Badaoui, Cyclostationary modelling of rotating machine vibration signals, Mech. Syst. Signal Process. 18 (6) (2004) $1285-1314$.

[11] J. Antoni, Cyclic spectral analysis of rolling-element bearing signals: facts and fictions, J. Sound Vib. 304 (3-5) (2007) 497-529. 
[12] P. Borghesani, P. Pennacchi, R. Ricci, S. Chatterton, Testing second order cyclostationarity in the squared envelope spectrum of non-white vibration signals, Mech. Syst. Signal Process. 40 (1) (2013) 38-55.

[13] W. Cioch, O. Knapik, J. Leskow, Finding a frequency signature for a cyclostationary signal with applications to wheel bearing diagnostics, Mech. Syst. Signal Process. 38 (1) (2013) 55-64 (condition monitoring of machines in non-stationary operations).

[14] J. Antoni, Cyclostationarity by examples, Mech. Syst. Signal Process. 23 (4) (2009) 987-1036.

[15] J. Antoni, D. Hanson, Detection of surface ships from interception of cyclostationary signature with the cyclic modulation coherence, IEEE J. Ocean. Eng. 37 (3) (2012) 478-493.

[16] P. Borghesani, The envelope-based cyclic periodogram, Mech. Syst. Signal Process. 58-59 (2015) 245-270.

[17] W.A. Brown, H.H. Loomis, Digital implementations of spectral correlation analyzers, IEEE Trans. Signal Process. 41 (2) (1993) $703-720$.

[18] R.S. Roberts, W.A. Brown, H.H. Loomis, Computationally efficient algorithms for cyclic spectral analysis, IEEE Signal Process. Mag. 8 (2) (1991) 38-49.

[19] R.B. Randall, J. Antoni, Rolling element bearing diagnostics - a tutorial, Mech. Syst. Signal Process. 25 (2) (2011) $485-520$.

[20] Bearing Data Center, Case Western Reserve Univ., Cleveland, OH [Online].

[21] W.A. Smith, R.B. Randall, Rolling element bearing diagnostics using the case western reserve university data: a benchmark study, Mech. Syst. Signal Process. 64-65 (2015) 100-131.

[22] P. Borghesani, P. Pennacchi, R. Randall, N. Sawalhi, R. Ricci, Application of cepstrum pre-whitening for the diagnosis of bearing faults under variable speed conditions, Mech. Syst. Signal Process. 36 (2) (2013) 370-384.

[23] J. Antoni, Fast computation of the kurtogram for the detection of transient faults, Mech. Syst. Signal Process. 21 (1) (2007) $108-124$.

[24] W. Bartelmus, R. Zimroz, A new feature for monitoring the condition of gearboxes in non-stationary operating conditions, Mech. Syst. Signal Process. 23 (5) (2009) 1528-1534.

[25] P. Borghesani, R. Ricci, S. Chatterton, P. Pennacchi, A new procedure for using envelope analysis for rolling element bearing diagnostics in variable operating conditions, Mech. Syst. Signal Process. 38 (1) (2013) 23-35 (condition monitoring of machines in non-stationary operations).

[26] J. Antoni, D. Abboud, S. Baudin, Cyclostationarity: Theory and Methods, Springer International Publishing, 2014, pp. 3-14 (Chapter Time-Angle Periodically Correlated Processes).

[27] A. Napolitano, Cyclostationarity: new trends and applications, Signal Process. 120 (2016) 385-408.

[28] G. D’Elia, Z. Daher, J. Antoni, A novel approach for the cyclo-non-stationary analysis of speed varying signals, in: ISMA2010 international conference on noise and vibration engineering, Leuven, 2010.

[29] S. Baudin, D. Rmond, J. Antoni, O. Sauvage, Non-intrusive rattle noise detection in non-stationary conditions by an angle/time cyclostationary approach, J. Sound Vib. 366 (2016) 501-513.

[30] D. Abboud, S. Baudin, J. Antoni, D. Rmond, M. Eltabach, O. Sauvage, The spectral analysis of cyclo-non-stationary signals, Mech. Syst. Signal Process. 75 (2016) 280-300.

[31] J. Antoni, M. Eltabach, A KIS solution for high fidelity interpolation and resampling of signals, Mech. Syst. Signal Process. 35 (1-2) (2013) 127-136.

[32] C. Mishra, A. Samantaray, G. Chakraborty, Rolling element bearing defect diagnosis under variable speed operation through angle synchronous averaging of wavelet de-noised estimate, Mech. Syst. Signal Process. 72-73 (2016) 206-222.

[33] Brillinger, Time Series: Data Analysis and Theory, SIAM: Society for Industrial and Applied Mathematics, 2001. 